CERN-PH-TH/2006-130

DCPT-06-98

IPPP-06-49

UWThPh-2006-17

hep-ph/0608065

\title{
CP asymmetries in chargino production and decay: the three-body decay case
}

\author{
A. Bartl ${ }^{1}$, H. Fraas ${ }^{2}$, S. Hesselbach ${ }^{2}$, K. Hohenwarter-Sodek ${ }^{1}$, \\ T. Kernreiter ${ }^{1}$, G. Moortgat-Pick ${ }^{3}$ \\ ${ }^{1}$ Institut für Theoretische Physik, Universität Wien, A-1090 Vienna, Austria \\ ${ }^{2}$ Institut für Theoretische Physik und Astrophysik, Universität Würzburg, \\ Am Hubland, D-97074 Würzburg, Germany \\ ${ }^{3}$ TH Division, Physics Department, CERN, CH-1211 Geneva 23, Switzerland
}

\begin{abstract}
We study CP violation in chargino production and decay in the Minimal Supersymmetric Standard Model (MSSM) with complex parameters at an $e^{+} e^{-}$linear collider with longitudinally polarized beams. We investigate CP-sensitive asymmetries by means of triple product correlations and study their dependence on the complex parameters $M_{1}$ and $\mu$. We give numerical predictions for the asymmetries and their measurability at the future International Linear Collider. Our results show that the CP asymmetries can be measured in a large region of the MSSM parameter space.
\end{abstract}




\section{Introduction}

In the Minimal Supersymmetric Standard Model (MSSM) [1] the supersymmetric partners of the gauge bosons and Higgs bosons with the same electric charge mix and form the neutralinos $\tilde{\chi}_{i}^{0}(i=1, \ldots, 4)$ and the charginos $\tilde{\chi}_{k}^{+}(k=1,2)$, as the neutral and charged mass eigenstates, respectively. The charginos and the neutralinos are of particular interest, as they will presumably be among the lightest supersymmetric (SUSY) particles. One of the main goals of the International Linear Collider (ILC) [2, 3] will be the determination of the underlying SUSY parameters. Those parameters that enter the neutralino/chargino system at tree level are the gaugino mass parameters $M_{1}$ and $M_{2}$, the higgsino mass parameter $\mu$, and the ratio of the vacuum expectation values of the Higgs fields, $\tan \beta$. Among these parameters $M_{1}$ and $\mu$ can be complex, while $M_{2}$ and $\tan \beta$ can be chosen real. In [4, 5] methods have been developed to determine the parameters in the neutralino and chargino system with and without $\mathrm{CP}$ violation by measurements of the neutralino and chargino masses and their production cross sections.

The phases $\phi_{\mu}$ and $\phi_{M_{1}}$ of $\mu$ and $M_{1}$ may be constrained or correlated by the experimental upper bounds on the electric dipole moments (EDMs). These constraints, however, are rather model-dependent [6]. While the restriction on the phase $\phi_{\mu}$, due to the electron EDM, is rather severe in a constrained MSSM with selectron masses of the order of $100 \mathrm{GeV}$ [7], it may disappear if lepton-flavour-violating terms in the MSSM Lagrangian are included [8]. Recently it has been pointed out that for large trilinear scalar couplings $A$ we can simultaneously fulfil the EDM constraints of electron, neutron, and of the atoms ${ }^{199} \mathrm{Hg}$ and ${ }^{205} \mathrm{Tl}$ where, at the same time, $\phi_{\mu} \sim O(1)[9]$. The size of the phase $\phi_{M_{1}}$, on the other hand, is less strongly restricted in the MSSM. Thus, the CP phases $\phi_{M_{1}}$ and $\phi_{\mu}$ can have a big influence on the production and decay of charginos and neutralinos at the ILC. In particular, they give rise to CP-sensitive observables that may be accessible at future collider experiments. Measurements of CP-sensitive observables are necessary to prove that $\mathrm{CP}$ is violated. Furthermore, only the inclusion of CP-sensitive observables allows us to deduce the underlying model parameters in an unambiguous way. In neutralino production with subsequent decay, CP-sensitive observables based on triple product correlations have been investigated in [10, 11, 12, 13. Also for the case of chargino production and decay, various CP-sensitive observables have been studied. Decay rate asymmetries in chargino decays have been studied in [14] and CP asymmetries in decay chains of sneutrinos involving charginos in [15]. CP-sensitive asymmetries based on triple product correlations have been analysed for the subsequent two-body decays $\tilde{\chi}_{j}^{-} \rightarrow \tilde{\chi}_{1}^{0} W^{-}[16]$ and $\tilde{\chi}_{j}^{-} \rightarrow \tilde{\nu}_{\ell} \ell^{-}$[17]. For the case of transverse $e^{ \pm}$beam polarization azimuthal asymmetries have been studied for the same two-body decays, showing a pronounced dependence on $\phi_{M_{1}}$ and $\phi_{\mu}$ [18]. In the present paper we extend previous investigations of $\mathrm{CP}$ violation in chargino production and decay to the case of chargino three-body decays.

We study the production processes

$$
e^{+} e^{-} \rightarrow \tilde{\chi}_{1}^{+} \tilde{\chi}_{k}^{-}, \quad k=1,2
$$

at a linear collider with longitudinal beam polarizations, and subsequent leptonic or 
hadronic three-body decays of the $\tilde{\chi}_{1}^{+}$,

$$
\tilde{\chi}_{1}^{+} \rightarrow \tilde{\chi}_{1}^{0} \nu \ell^{+}, \quad \ell=e, \mu
$$

and

$$
\tilde{\chi}_{1}^{+} \rightarrow \tilde{\chi}_{1}^{0} \bar{s} c
$$

where we assume that the momenta $\vec{p}_{\tilde{\chi}_{1}^{+}}, \vec{p}_{\ell}, \vec{p}_{c}$ and $\vec{p}_{s}$ of the associated particles can be measured or reconstructed. We study two T-odd observables based on triple product correlations of momentum vectors:

$$
\begin{aligned}
& \mathcal{T}_{\ell}=\vec{p}_{\ell^{+}} \cdot\left(\vec{p}_{e^{-}} \times \vec{p}_{\tilde{\chi}_{1}^{+}}\right), \\
& \mathcal{T}_{q}=\vec{p}_{\bar{s}} \cdot\left(\vec{p}_{c} \times \vec{p}_{e^{-}}\right) .
\end{aligned}
$$

The triple product $\mathcal{T}_{\ell}$, Eq. (4) , relates momenta of initial, intermediate and final particles, whereas $\mathcal{T}_{q}$, Eq. (5), uses only momenta from the initial and final states. Therefore, both triple products depend in a different way on the production and decay processes.

The triple product $\mathcal{T}_{\ell}$, Eq. (4), involves the momentum of the decay lepton that usually can be very accurately measured. However, the momentum of the chargino has to be reconstructed with information from the decay of the second chargino $\tilde{\chi}_{k}^{-}$[13]. For the triple product $\mathcal{T}_{q}$, Eq. (5), it is necessary to identify the $c$-quark, which is expected to be possible with reasonable efficiency and purity [19, 20, 21]. To derive the CP-violating asymmetry also the charge of the $c$-quark has to be detected, which can be done with specific vertex detectors [19, 22, 23]. The corresponding T-odd asymmetries are defined by

$$
A_{T}\left(\mathcal{T}_{\ell, q}\right)=\frac{N\left[\mathcal{T}_{\ell, q}>0\right]-N\left[\mathcal{T}_{\ell, q}<0\right]}{N\left[\mathcal{T}_{\ell, q}>0\right]+N\left[\mathcal{T}_{\ell, q}<0\right]}
$$

where $N\left[\mathcal{T}_{\ell, q}>(<) 0\right]$ is the number of events for which $\mathcal{T}_{\ell, q}>(<) 0$.

Finally we recall that a non-zero value of the T-odd asymmetries does not immediately imply that the CP symmetry is violated since final-state interactions give rise (although only at the one-loop level) to the same asymmetries. However, a genuine signal of CP violation can be obtained when one combines $A_{T}\left(\mathcal{T}_{\ell, q}\right)$ with the corresponding asymmetry $\bar{A}_{T}\left(\mathcal{T}_{\ell, q}\right)$ for the charge-conjugated processes. Then in the CP asymmetries

$$
A_{\mathrm{CP}}\left(\mathcal{T}_{\ell, q}\right)=\frac{A_{T}\left(\mathcal{T}_{\ell, q}\right)-\bar{A}_{T}\left(\mathcal{T}_{\ell, q}\right)}{2}
$$

the effect of final-state interactions cancels out.

The paper is organized as follows. In Section 2 we briefly recall the formalism, which we use to calculate the cross sections and the CP asymmetries. We present our numerical results in Section 3. Finally, we summarize and conclude in Section 4. 


\section{Cross section and CP asymmetries}

The chargino production process (11) proceeds via $\gamma$ and $Z^{0}$ exchange in the $s$-channel and via $\tilde{\nu}_{e}$ exchange in the $t$-channel (Fig. (1). The decay processes (2) and (3) contain contributions from $W^{+}, \tilde{\ell}_{L}(\ell=e, \mu)$ and $\tilde{\nu}_{\ell}$ exchange in the leptonic case and from $W^{+}$, $\tilde{c}_{L}$ and $\tilde{s}_{L}$ exchange in the hadronic case (Fig. 22). The interaction Lagrangians for these processes can be found, for instance, in [24].

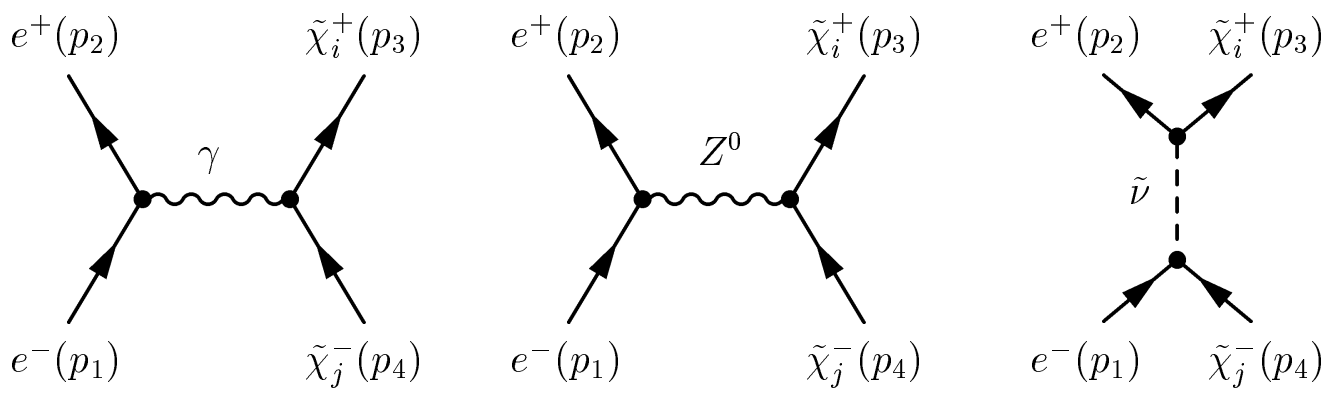

Figure 1: Feynman diagrams of the production process $e^{+} e^{-} \rightarrow \tilde{\chi}_{i}^{+} \tilde{\chi}_{j}^{-}$.
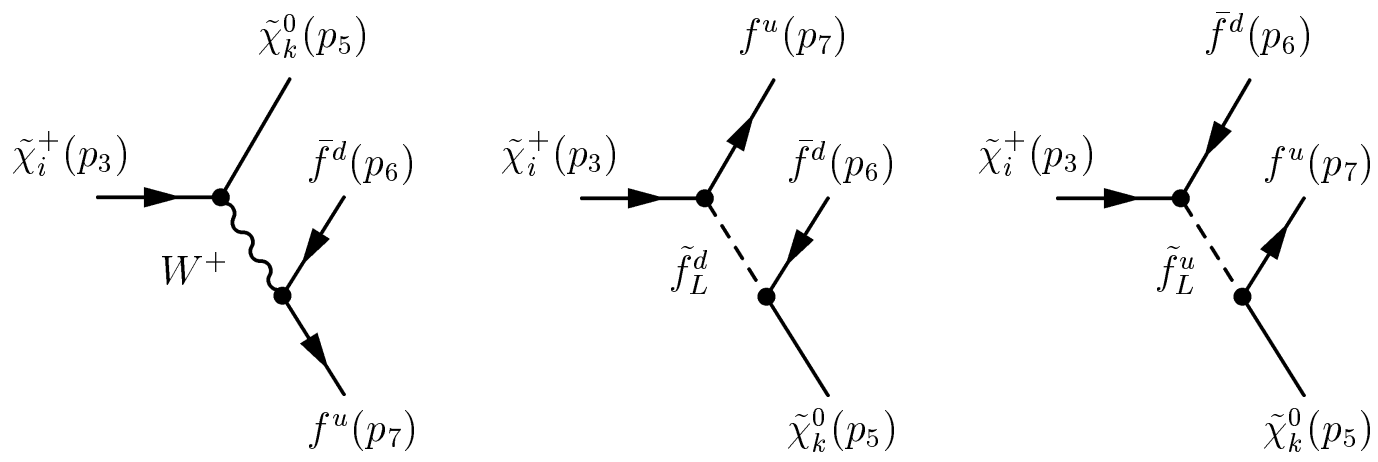

Figure 2: Feynman diagrams of the three-body decay $\tilde{\chi}_{i}^{+} \rightarrow \tilde{\chi}_{k}^{0} \bar{f}^{d} f^{u}$, where $f^{d}=e, \mu, s$ and $f^{u}=\nu_{e}, \nu_{\mu}, c$.

\subsection{Cross section}

For the calculation of the squared amplitude of the whole process $e^{+} e^{-} \rightarrow \tilde{\chi}_{i}^{+} \tilde{\chi}_{j}^{-} \rightarrow$ $\tilde{\chi}_{1}^{0} \bar{f}^{d} f^{u} \tilde{\chi}_{j}^{-}$, we use the spin-density matrix formalism [24, 25]. The squared amplitude can then be written as

$$
|T|^{2}=2\left|\Delta\left(\tilde{\chi}_{i}^{+}\right)\right|^{2} \sum_{\lambda_{i}, \lambda_{i}^{\prime}} \rho_{P}{ }^{\lambda_{i} \lambda_{i}^{\prime}} \rho_{D \lambda_{i}^{\prime} \lambda_{i}},
$$

with the propagator $\Delta\left(\tilde{\chi}_{i}^{+}\right)=1 /\left[p_{\tilde{\chi}_{i}^{+}}^{2}-m_{i}^{2}+i m_{i} \Gamma_{i}\right]$. Here, $\lambda_{i}, \lambda_{i}^{\prime}, m_{i}, \Gamma_{i}$ denote the helicities, masses and widths of the chargino $\tilde{\chi}_{i}^{+}$. The factor 2 in Eq. (8) is due to the summation over the helicities of chargino $\tilde{\chi}_{j}^{-}$, whose decay is not considered. The squared 
amplitude is composed of the unnormalized spin-density matrices $\rho_{P}$ for the production and $\rho_{D}$ for the decay, which carry the helicity indices $\lambda_{i}, \lambda_{i}^{\prime}$ of the chargino $\tilde{\chi}_{i}^{+}$. Introducing a set of polarization basis 4 -vectors $s_{\chi_{i}}^{a}(a=1,2,3)$ for the charginos $\tilde{\chi}_{i}^{+}$, where $s_{\chi_{i}}^{3}$ describes the longitudinal polarization and $s_{\chi_{i}}^{1}, s_{\chi_{i}}^{2}$ the transverse polarization in and perpendicular to the production plane, respectively, and which fulfil the orthonormality relations $s_{\chi_{i}}^{a} \cdot s_{\chi_{i}}^{b}=-\delta^{a b}$ and $s_{\chi_{i}}^{a} \cdot p_{\chi_{i}}=0$, the density matrices can be expanded in terms of the Pauli matrices:

$$
\begin{aligned}
& \rho_{P}^{\lambda_{i} \lambda_{i}^{\prime}}=\delta_{\lambda_{i} \lambda_{i}^{\prime}} P+\sum_{a=1}^{3} \sigma_{\lambda_{i} \lambda_{i}^{\prime}}^{a} \Sigma_{P}^{a}, \\
& \rho_{D \lambda_{i}^{\prime} \lambda_{i}}=\delta_{\lambda_{i}^{\prime} \lambda_{i}} D+\sum_{a=1}^{3} \sigma_{\lambda_{i}^{\prime} \lambda_{i}}^{a} \Sigma_{D}^{a} .
\end{aligned}
$$

Then the squared amplitude is given by

$$
|T|^{2}=4\left|\Delta\left(\tilde{\chi}_{i}^{+}\right)\right|^{2}\left\{P\left(\tilde{\chi}_{i}^{+} \tilde{\chi}_{j}^{-}\right) D\left(\tilde{\chi}_{i}^{+}\right)+\sum_{a=1}^{3} \Sigma_{P}^{a}\left(\tilde{\chi}_{i}^{+}\right) \Sigma_{D}^{a}\left(\tilde{\chi}_{i}^{+}\right)\right\},
$$

where $P\left(\tilde{\chi}_{i}^{+} \tilde{\chi}_{j}^{-}\right)$and $D\left(\tilde{\chi}_{i}^{+}\right)$are those parts of the spin density production and decay matrices, that are independent of the polarization of the charginos. The contributions $\Sigma_{P}^{a}\left(\tilde{\chi}_{i}^{+}\right)$and $\Sigma_{D}^{a}\left(\tilde{\chi}_{i}^{+}\right)$depend on the polarization vector $s^{a}$ of the decaying chargino $\tilde{\chi}_{i}^{+}$. The full expressions for the quantities $P\left(\tilde{\chi}_{i}^{+} \tilde{\chi}_{j}^{-}\right), D\left(\tilde{\chi}_{i}^{+}\right), \Sigma_{P}^{a}\left(\tilde{\chi}_{i}^{+}\right)$and $\Sigma_{D}^{a}\left(\tilde{\chi}_{i}^{+}\right)$can be found in [24]. Finally, the differential cross section is given by

$$
d \sigma=\frac{1}{8 E_{b}^{2}}|T|^{2}(2 \pi)^{4} \delta^{4}\left(p_{1}+p_{2}-\sum_{i=4}^{7} p_{i}\right) d \operatorname{lips}\left(p_{3} \cdots p_{7}\right)
$$

where $E_{b}$ is the beam energy and $d \operatorname{lips}\left(p_{3} \cdots p_{7}\right)$ is the Lorentz-invariant phase-space element.

\subsection{CP asymmetries}

The T-odd asymmetries defined in Eq. (6) are calculated as

$$
A_{T}\left(\mathcal{T}_{\ell, q}\right)=\frac{\int \operatorname{sign}\left\{\mathcal{T}_{\ell, q}\right\}|T|^{2} d \text { lips }}{\int|T|^{2} d \text { lips }}
$$

where we weight the sign of the triple product correlations in Eqs. (4) and (5) with the associated squared amplitude. Since in the numerator $\int \operatorname{sign}\left\{\mathcal{T}_{\ell, q}\right\} P\left(\tilde{\chi}_{i}^{+} \tilde{\chi}_{j}^{-}\right) D\left(\tilde{\chi}_{i}^{+}\right) d \operatorname{lips}=$ 0 and in the denominator $\int \Sigma_{P}^{a}\left(\tilde{\chi}_{i}^{+}\right) \Sigma_{D}^{a}\left(\tilde{\chi}_{i}^{+}\right) d$ lips $=0$, we obtain by inserting the squared amplitude, Eq. (11), into Eq. (13):

$$
A_{T}\left(\mathcal{T}_{\ell, q}\right)=\frac{\int \operatorname{sign}\left\{\mathcal{T}_{\ell, q}\right\} \Sigma_{P}^{a}\left(\tilde{\chi}_{i}^{+}\right) \Sigma_{D}^{a}\left(\tilde{\chi}_{i}^{+}\right) d \operatorname{lips}}{\int P\left(\tilde{\chi}_{i}^{+} \tilde{\chi}_{j}^{-}\right) D\left(\tilde{\chi}_{i}^{+}\right) d \operatorname{lips}}
$$


We split $\Sigma_{P}^{a}\left(\tilde{\chi}_{i}^{+}\right)$and $\Sigma_{D}^{a}\left(\tilde{\chi}_{i}^{+}\right)$into the T-odd terms $\Sigma_{P}^{a, \mathrm{O}}\left(\tilde{\chi}_{i}^{+}\right)$and $\Sigma_{D}^{a, \mathrm{O}}\left(\tilde{\chi}_{i}^{+}\right)$, which contain the respective triple product, and T-even terms $\Sigma_{P}^{a, \mathrm{E}}\left(\tilde{\chi}_{i}^{+}\right)$and $\Sigma_{D}^{a, \mathrm{E}}\left(\tilde{\chi}_{i}^{+}\right)$without triple products:

$$
\Sigma_{P}^{a}\left(\tilde{\chi}_{i}^{+}\right)=\Sigma_{P}^{a, \mathrm{O}}\left(\tilde{\chi}_{i}^{+}\right)+\Sigma_{P}^{a, \mathrm{E}}\left(\tilde{\chi}_{i}^{+}\right), \quad \Sigma_{D}^{a}\left(\tilde{\chi}_{i}^{+}\right)=\Sigma_{D}^{a, \mathrm{O}}\left(\tilde{\chi}_{i}^{+}\right)+\Sigma_{D}^{a, \mathrm{E}}\left(\tilde{\chi}_{i}^{+}\right) .
$$

The terms of $|T|^{2}$, Eq. (11), which contribute to the numerator of $A_{T}$ are

$$
\Sigma_{P}^{a, \mathrm{O}}\left(\tilde{\chi}_{i}^{+}\right) \Sigma_{D}^{a, \mathrm{E}}\left(\tilde{\chi}_{i}^{+}\right)+\Sigma_{P}^{a, \mathrm{E}}\left(\tilde{\chi}_{i}^{+}\right) \Sigma_{D}^{a, \mathrm{O}}\left(\tilde{\chi}_{i}^{+}\right),
$$

where the first (second) term is sensitive to the CP phases in the production (decay) process of the chargino $\tilde{\chi}_{i}^{+}$. The explicit expressions for the T-odd and T-even contributions in Eq. (16) are given in Appendix A. (The analytical expressions of the quantities $P\left(\tilde{\chi}_{i}^{+} \tilde{\chi}_{j}^{-}\right)$and $D\left(\tilde{\chi}_{i}^{+}\right)$can be found in [24].) With $A_{T}\left(\mathcal{T}_{\ell, q}\right)$ we calculate the corresponding $\mathrm{CP}$ asymmetries $A_{\mathrm{CP}}\left(\mathcal{T}_{\ell, q}\right)$ according to Eq. (77).

\section{$3 \quad$ Numerical results}

In this section we give numerical results for the $\mathrm{CP}$ asymmetries $A_{\mathrm{CP}}\left(\mathcal{T}_{\ell, q}\right)$, Eq. (7), for the reactions (11), (2), (3), at an $e^{+} e^{-}$linear collider with centre-of-mass energy $\sqrt{s}=500 \mathrm{GeV}$ and longitudinally polarized beams. We analyse the hadronic decay $\tilde{\chi}_{1}^{+} \rightarrow \tilde{\chi}_{1}^{0} \bar{s} c$ and the leptonic decays $\tilde{\chi}_{1}^{+} \rightarrow \tilde{\chi}_{1}^{0} \ell^{+} \nu, \ell=e, \mu$. To this end, we consider three scenarios (see Tables 1, 22 and 3) for which $m_{\tilde{\chi}_{1}^{+}}<m_{W}+m_{\tilde{\chi}_{1}^{0}}$ and $m_{\tilde{\chi}_{1}^{+}}<m_{\tilde{f}_{L}^{u, d}}$ to rule out two-body decays of $\tilde{\chi}_{1}^{+}$. The chargino decay widths and branching ratios have been calculated with the computer program SPheno [26].

The statistical significance to which $A_{\mathrm{CP}}$ can be determined to be non-zero can be estimated in the following way: Assuming that the statistical errors of $A_{T}$ [27] and $\bar{A}_{T}$ are independent of each other, the errors of $A_{T}$ and $\bar{A}_{T}$ are added in quadrature. The absolute error of $A_{\mathrm{CP}}$ is then given by

$$
\Delta A_{\mathrm{CP}}=\mathcal{N}_{\sigma} \frac{\sqrt{1-A_{\mathrm{CP}}^{2}}}{\sqrt{2 \sigma \mathcal{L}_{\text {int }}}},
$$

where $\mathcal{N}_{\sigma}$ denotes the respective number of standard deviations, $\sigma=\sigma\left(e^{+} e^{-} \rightarrow \tilde{\chi}_{1}^{+} \tilde{\chi}_{j}^{-}\right)$. $B\left(\tilde{\chi}_{1}^{+} \rightarrow \tilde{\chi}_{1}^{0} f^{\prime} \bar{f}\right)$ being the corresponding cross section of the combined production and decay processes and $\mathcal{L}_{\text {int }}$ is the integrated luminosity, where we assume $\mathcal{L}_{\text {int }}=500 \mathrm{fb}^{-1}$ in the theoretical estimates below. For $A_{\mathrm{CP}} \lesssim 10 \%$, i.e. $A_{\mathrm{CP}}^{2} \lesssim 0.01$, it is $\Delta A_{\mathrm{CP}}=$ $\mathcal{N}_{\sigma} / \sqrt{2 \sigma \mathcal{L}_{\text {int }}}$ in good approximation. If we require $A_{\mathrm{CP}}>\Delta A_{\mathrm{CP}}$ for $A_{\mathrm{CP}}$ to be measurable we obtain

$$
\mathcal{N}_{\sigma}=\sqrt{2 A_{\mathrm{CP}}^{2} \sigma \mathcal{L}_{\text {int }}}
$$




\begin{tabular}{|c|c|c|c|c|c|c|c|c|c|}
\hline & $\phi_{\mu}$ & $\phi_{M_{1}}$ & $m_{\tilde{\chi}_{1}^{0}}$ & $m_{\tilde{\chi}_{2}^{0}}$ & $m_{\tilde{\chi}_{3}^{0}}$ & $m_{\tilde{\chi}_{4}^{0}}$ & $m_{\tilde{\chi}_{1}^{ \pm}}$ & $m_{\tilde{\chi}_{2}^{ \pm}}$ \\
\hline & & $\overline{c 0}$ & $\overline{0}$ & 119.3 & $\overline{184.3}$ & 205.9 & 322.7 & 166.2 & 322.1 \\
\hline \multicolumn{2}{|c|}{ scenario A } & 0 & $\frac{\pi}{2}$ & 126.3 & 176.0 & 210.0 & 323.0 & 166.2 & 322.1 \\
\hline$\overline{\overline{M_{2}}}$ & 280 & 0 & $\pi$ & 135.3 & 166.7 & 213.0 & 321.3 & 166.2 & 322.1 \\
\hline$|\mu|$ & 200 & \multirow{3}{*}{$\begin{array}{l}\frac{\pi}{2} \\
\frac{\pi}{2} \\
\frac{\pi}{2}\end{array}$} & \multirow{3}{*}{$\begin{array}{l}\frac{\pi}{2} \\
\pi\end{array}$} & \multirow{3}{*}{$\begin{array}{l}127.6 \\
134.8 \\
129.6\end{array}$} & \multirow{3}{*}{$\begin{array}{l}187.4 \\
178.3 \\
176.7\end{array}$} & \multirow{3}{*}{$\begin{array}{l}208.5 \\
213.0 \\
217.8\end{array}$} & 316.0 & 177.4 & 316.0 \\
\hline $\tan \beta$ & 5 & & & & & & 315.3 & 177.4 & \multirow{2}{*}{$\begin{array}{l}316.0 \\
316.0\end{array}$} \\
\hline$m_{\tilde{\nu}}$ & 250 & & & & & & 315.0 & 177.4 & \\
\hline \multirow[t]{3}{*}{$m_{\tilde{u}_{L}}$} & 500 & \multirow{3}{*}{$\begin{array}{l}\pi \\
\pi \\
\pi\end{array}$} & \multirow[b]{3}{*}{$\pi$} & \multirow{3}{*}{$\begin{array}{l}134.6 \\
130.3 \\
126.0\end{array}$} & \multirow{3}{*}{$\begin{array}{l}190.4 \\
186.0 \\
183.3\end{array}$} & \multirow{3}{*}{$\begin{array}{l}212.9 \\
219.9 \\
225.1\end{array}$} & 308.2 & 189.2 & \multirow{3}{*}{$\begin{array}{l}309.1 \\
309.1 \\
309.1\end{array}$} \\
\hline & & & & & & & 307.9 & 189.2 & \\
\hline & & & & & & & 307.5 & 189.2 & \\
\hline
\end{tabular}

Table 1: Input parameters $M_{2},|\mu|, \tan \beta, m_{\tilde{\nu}}$ and $m_{\tilde{u}_{L}}=m_{\tilde{c}_{L}}$ and neutralino and chargino masses for scenario A for different values of $\phi_{\mu}$ and $\phi_{M_{1}} .\left|M_{1}\right|$ is fixed by the GUT-inspired relation $\left|M_{1}\right|=5 / 3 \tan ^{2} \theta_{W} M_{2}$ and the masses of the down-type sfermions by the $\mathrm{SU}(2)$ relation. All masses are given in $\mathrm{GeV}$.

\subsection{CP asymmetry for $\tilde{\chi}_{1}^{+} \tilde{\chi}_{1}^{-}$production and $\tilde{\chi}_{1}^{+}$decay}

In the case of pair production, $e^{+} e^{-} \rightarrow \tilde{\chi}_{1}^{+} \tilde{\chi}_{1}^{-}$, only CP-violating couplings from the decay (second term in Eq. (16)) can give rise to a CP-violating effect, because in the production (first term in Eq. (16)) only the absolute squares of the couplings enter. Thus, the CP asymmetry $A_{\mathrm{CP}}\left(\mathcal{T}_{q}\right)$ is sensitive to the $\mathrm{CP}$ violation in the decay, due to the phases of $\mu$ and $M_{1}$. Fig. 3 (a) shows the asymmetry $A_{\mathrm{CP}}\left(\mathcal{T}_{q}\right)$ as a function of the phase $\phi_{M_{1}}$ for scenario A (see Table 1) for $\phi_{\mu}=0$. The masses of the squarks are chosen to be $m_{\tilde{c}}=500 \mathrm{GeV}$ and $m_{\tilde{s}}=505.9 \mathrm{GeV}$. The centre-of-mass energy $\sqrt{s}=500 \mathrm{GeV}$ and the two sets of longitudinal $e^{ \pm}$beam polarizations are fixed in our study at $\left(P_{e^{-}}, P_{e^{+}}\right)=(+0.8,-0.6)$ and $\left(P_{e^{-}}, P_{e^{+}}\right)=(-0.8,+0.6)$. The $\mathrm{CP}$ asymmetry reaches its largest value of about $3.7 \%$ for $\left(P_{e^{-}}, P_{e^{+}}\right)=(-0.8,+0.6)$ at $\phi_{M_{1}}=1.2 \pi$. Note that the asymmetry changes its sign for the two different sets of beam polarization due to the prefactor (Eq. (25), Appendix A) which depends on the longitudinal beam polarization. Note further that the asymmetry does not have its largest absolute value for $\phi_{M_{1}}=0.5 \pi, 1.5 \pi$. This behaviour is due to a complex interplay of the $\phi_{M_{1}}$ dependence of the numerator and denominator of the asymmetry in Eq. (14). In Fig. 3 (b) we show the dependence of the CP asymmetry $A_{\mathrm{CP}}\left(\mathcal{T}_{q}\right)$ on $\phi_{\mu}$ for the same scenario taking $\phi_{M_{1}}=\pi$. The maximum value of about $4.6 \%$ of $A_{\mathrm{CP}}\left(\mathcal{T}_{q}\right)$ is reached at $\phi_{\mu}=0.3 \pi$ for $\left(P_{e^{-}}, P_{e^{+}}\right)=(-0.8,+0.6)$.

In Fig. (4) (a) and (b) the contours of the CP asymmetry $A_{\mathrm{CP}}\left(\mathcal{T}_{q}\right)$, Eq. (7), are shown in the $M_{2^{-}}|\mu|$ plane. The other MSSM parameters are chosen to be $\tan \beta=5, m_{\tilde{\nu}}=250 \mathrm{GeV}$, $m_{\tilde{c}}=500 \mathrm{GeV}, m_{\tilde{s}}=505.9 \mathrm{GeV},\left|M_{1}\right|=5 / 3 \tan ^{2} \theta_{W} M_{2}, \phi_{M_{1}}=0.5 \pi$ and $\phi_{\mu}=0$. For both polarization configurations, $\left(P_{e^{-}}, P_{e^{+}}\right)=(-0.8,+0.6)$ and $\left(P_{e^{-}}, P_{e^{+}}\right)=(+0.8,-0.6)$, the absolute value of $A_{\mathrm{CP}}\left(\mathcal{T}_{q}\right)$ is largest in the region $|\mu| \approx 260 \mathrm{GeV}$ and $M_{2} \approx 360 \mathrm{GeV}$ with asymmetries of about $-5 \%(4 \%)$ for $\left(P_{e^{-}}, P_{e^{+}}\right)=(-0.8,+0.6)((+0.8,-0.6))$. The main contributions to the numerator of the asymmetry are due to the $W^{+}-\tilde{s}_{L}$ and $W^{+}-\tilde{c}_{L}$ interference terms.

Figs. 4 (c) and (d) show the contours of the corresponding number of standard de- 

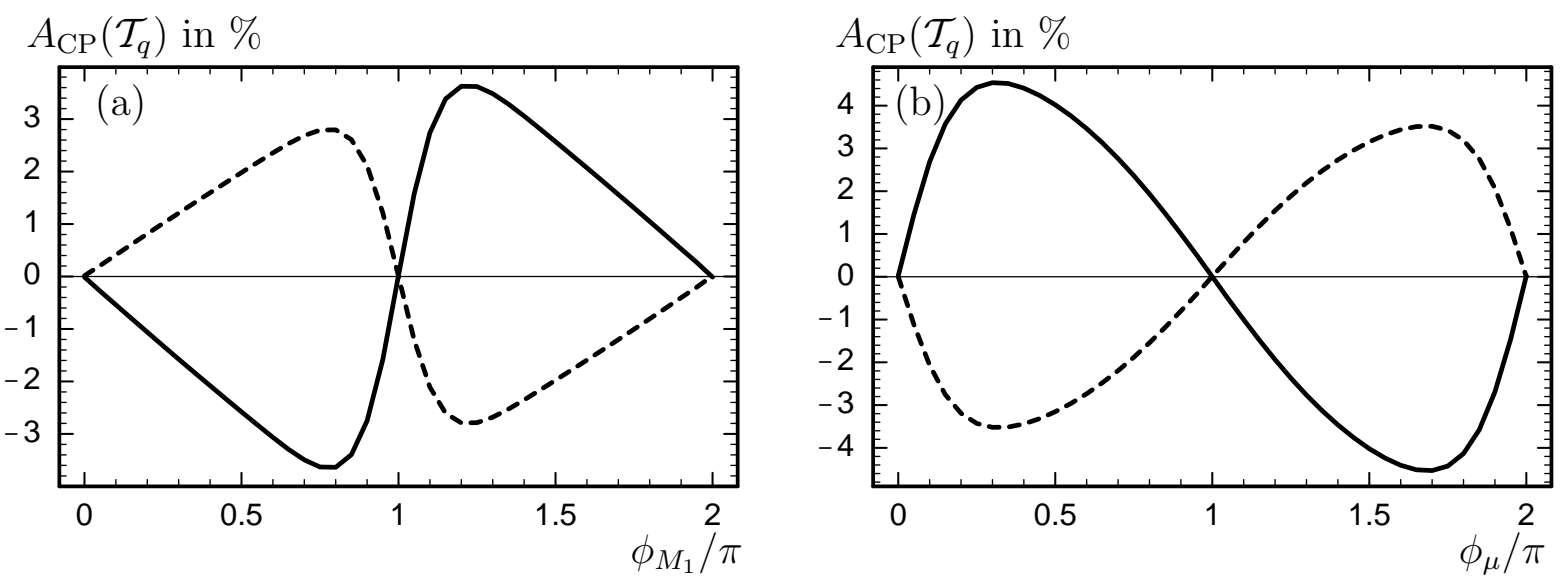

Figure 3: CP asymmetry $A_{\mathrm{CP}}\left(\mathcal{T}_{q}\right)$, Eq. (7), for $e^{+} e^{-} \rightarrow \tilde{\chi}_{1}^{+} \tilde{\chi}_{1}^{-}$with subsequent decay $\tilde{\chi}_{1}^{+} \rightarrow \tilde{\chi}_{1}^{0} \bar{s} c$ for the parameters defined in Table 1 (a) with $\phi_{\mu}=0$ and (b) with $\phi_{M_{1}}=\pi$, for $\sqrt{s}=500 \mathrm{GeV}$ and for the beam polarizations $\left(P_{e^{-}}, P_{e^{+}}\right)=(-0.8,+0.6)$ (solid), $\left(P_{e^{-}}, P_{e^{+}}\right)=(+0.8,-0.6)$ (dashed).

viations $\mathcal{N}_{\sigma}$ for an integrated luminosity $\mathcal{L}_{\text {int }}=500 \mathrm{fb}^{-1}$ in the $M_{2^{-}}|\mu|$ plane. Quite generally, the choice $\left(P_{e^{-}}, P_{e^{+}}\right)=(-0.8,+0.6)$ for longitudinal beam polarizations yields better results than $\left(P_{e^{-}}, P_{e^{+}}\right)=(+0.8,-0.6)$, because it enhances the sneutrino-exchange contribution to the production cross section. It is interesting to note that the asymmetry $A_{\mathrm{CP}}\left(\mathcal{T}_{q}\right)$ is measurable with a $5 \sigma$ significance in a large region of the parameter space.

Our numerical results for the number of standard deviations $\mathcal{N}_{\sigma}$ shown in Figs 4 (c), (d) and Figs. 6 (c), (d) below, do not include the influence of c-tagging, which is necessary for a measurement of $A_{\mathrm{CP}}\left(\mathcal{T}_{q}\right)$. Now we want to estimate how the detection rates are expected to be modified if the effects of c-tagging are also taken into account. Identifying the c-quark can be accomplished with the help of vertex detectors [19]. It has been shown in [20] that c-quarks will be identified with an efficiency of about $50 \%$ at a purity of $80 \%$ in $Z^{0}$ decays in $e^{+} e^{-} \rightarrow q \bar{q}$ at $\sqrt{s}=500 \mathrm{GeV}$. Accordingly, the number of standard deviations shown in Figs. 4 (c), (d) and Figs. 6 (c), (d) below, for the measurement of the $\mathrm{CP}$ asymmetry $A_{\mathrm{CP}}\left(\mathcal{T}_{q}\right)$ is expected to be reduced by a factor of about 0.57 . We note that the purity of c-jets in chargino and $W$ decays is presumably larger [27], since in this case fewer non-charm jets appear (the ratio of true charm to non-charm jets is approximately $1 / 3$ for $\mathrm{W}$ decays as compared to approximately $1 / 5$ for $\mathrm{Z}$ decays [29]). For measuring the $\mathrm{CP}$ asymmetry $A_{\mathrm{CP}}\left(\mathcal{T}_{q}\right)$ it is also necessary to distinguish the c-quark in the decay $\tilde{\chi}_{1}^{+} \rightarrow \tilde{\chi}_{1}^{0} c \bar{s}$ from its antiquark $\bar{c}$. This can be achieved with very good precision in the semi-leptonic decays of the charmed hadrons. For the majority of c-jets it can also be accomplished by the reconstruction of the vertex charge in the cases where the charmed hadrons decay non-leptonically [19, 22, 23]. The electric charge of the c-quark can also be indirectly identified in the cases where the second chargino, $\tilde{\chi}_{1}^{-}$, decays leptonically or where the sign of the charge of the $\bar{c}$-jet in the decay $\tilde{\chi}_{1}^{-} \rightarrow \tilde{\chi}_{1}^{0} \bar{c} s$ is determined. 

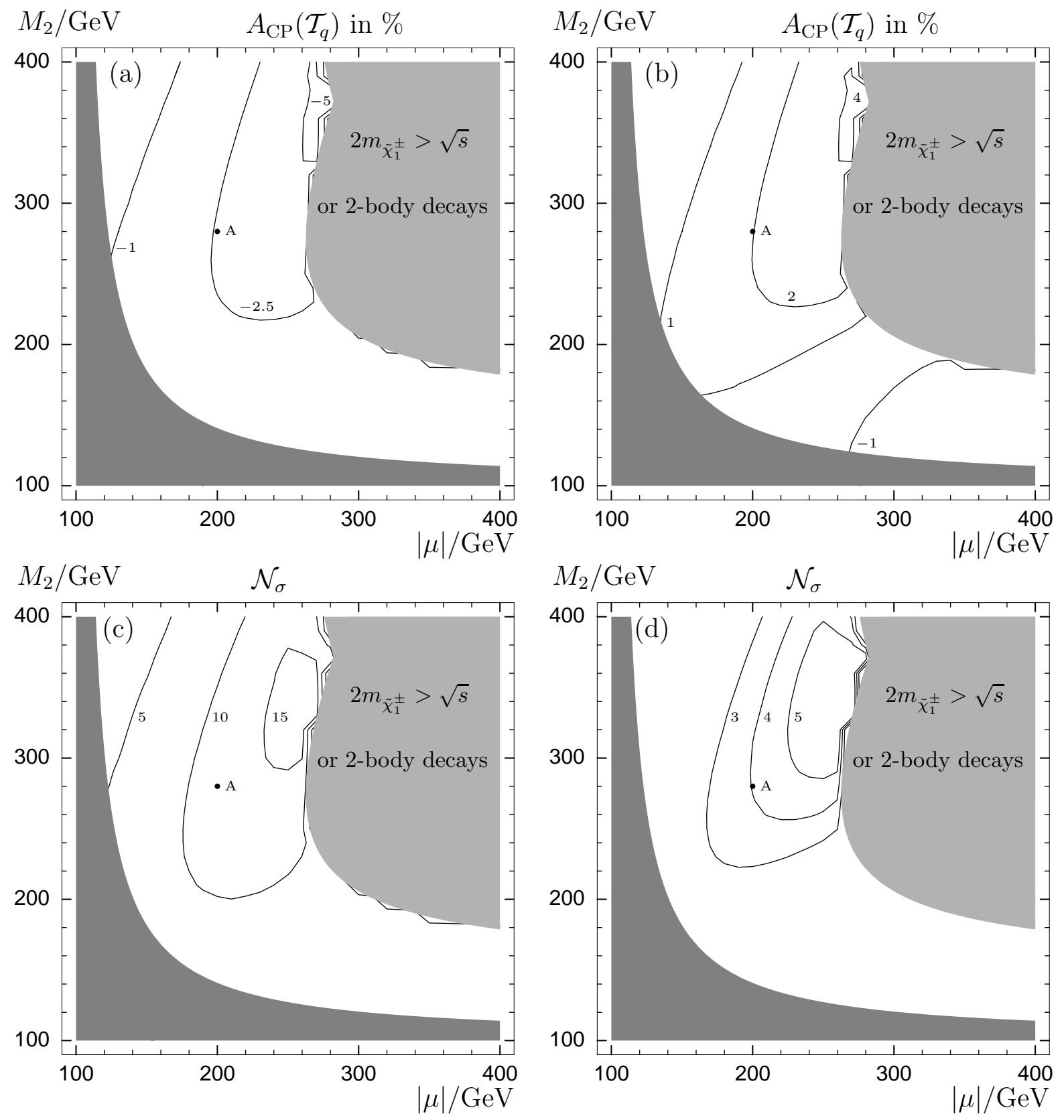

Figure 4: (a), (b) Contours of the CP asymmetry $A_{\mathrm{CP}}\left(\mathcal{T}_{q}\right)$, Eq. (17), in \% for $e^{+} e^{-} \rightarrow$ $\tilde{\chi}_{1}^{+} \tilde{\chi}_{1}^{-}$at $\sqrt{s}=500 \mathrm{GeV}$ with subsequent decay $\tilde{\chi}_{1}^{+} \rightarrow \tilde{\chi}_{1}^{0} \bar{s} c$ and $(\mathrm{c})$, (d) contours of the number of standard deviations $\mathcal{N}_{\sigma}$, Eq. (18), for an integrated luminosity $\mathcal{L}_{\text {int }}=500 \mathrm{fb}^{-1}$, respectively. The parameters are $\tan \beta=5, m_{\tilde{\nu}}=250 \mathrm{GeV}, m_{\tilde{c}}=500 \mathrm{GeV}, m_{\tilde{s}}=$ $505.9 \mathrm{GeV},\left|M_{1}\right| / M_{2}=5 / 3 \tan ^{2} \theta_{W}, \phi_{M_{1}}=0.5 \pi, \phi_{\mu}=0$. The beam polarizations are in (a), (c), $\left(P_{e^{-}}, P_{e^{+}}\right)=(-0.8,+0.6)$ and in (b), (d), $\left(P_{e^{-}}, P_{e^{+}}\right)=(+0.8,-0.6)$. The point marks the scenario A, defined in Table 1. In the dark-shaded area is $m_{\tilde{\chi}_{1}^{ \pm}}<103.5 \mathrm{GeV}$, excluded by LEP [28]. The light-shaded area shows the region that either is kinematically not accessible or in which the three-body decay is strongly suppressed because $m_{\tilde{\chi}_{1}^{+}}>$ $m_{W}+m_{\tilde{\chi}_{1}^{0}}$. 


\begin{tabular}{|c|c|c|c|c|c|c|c|c|c|}
\hline & $\phi_{\mu}$ & $\phi_{M_{1}}$ & $m_{\tilde{\chi}_{1}^{0}}$ & $m_{\tilde{\chi}_{2}^{0}}$ & $m_{\tilde{\chi}_{3}^{0}}$ & $m_{\tilde{\chi}_{4}^{0}}$ & $m_{\tilde{\chi}_{1}^{ \pm}}$ & $m_{\tilde{\chi}_{2}^{ \pm}}$ \\
\hline & & $\overline{00}$ & 0 & $\overline{70.6}$ & $\overline{132.5}$ & 325.7 & $\overline{347.8}$ & $\overline{~ 131.0}$ & $\overline{347.4}$ \\
\hline \multicolumn{2}{|c|}{ scenario B } & 0 & $\frac{\pi}{2}$ & 73.4 & 132.0 & 326.2 & 347.0 & 131.0 & 347.4 \\
\hline 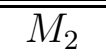 & 150 & 0 & $\pi$ & 76.1 & 131.4 & 326.6 & 346.2 & 131.0 & 347.4 \\
\hline$|\mu|$ & 320 & \multirow{3}{*}{$\begin{array}{l}\frac{\pi}{2} \\
\frac{\pi}{2} \\
\frac{\pi}{2}\end{array}$} & \multirow[b]{3}{*}{$\pi$} & \multirow{3}{*}{$\begin{array}{l}73.7 \\
76.1 \\
73.5\end{array}$} & 140.1 & 327.3 & 342.7 & 139.7 & \multirow{3}{*}{$\begin{array}{l}344.0 \\
344.0 \\
344.0\end{array}$} \\
\hline $\tan \beta$ & 5 & & & & 139.8 & 328.0 & 341.6 & 139.7 & \\
\hline$m_{\tilde{\nu}}$ & 250 & & & & 140.0 & 328.1 & 341.9 & 139.7 & \\
\hline$m_{\tilde{u}_{L}}$ & 500 & $\pi$ & \multirow{3}{*}{$\begin{array}{l}0 \\
\frac{\pi}{2} \\
\pi\end{array}$} & \multirow{3}{*}{$\begin{array}{l}76.1 \\
73.8 \\
71.5\end{array}$} & 148.0 & 332.5 & 333.6 & 148.3 & \multirow{3}{*}{$\begin{array}{l}340.3 \\
340.3 \\
340.3\end{array}$} \\
\hline & & $\pi$ & & & 148.0 & 332.7 & 334.0 & 148.3 & \\
\hline & & $\pi$ & & & 148.0 & 332.8 & 334.4 & 148.3 & \\
\hline
\end{tabular}

Table 2: Input parameters $M_{2},|\mu|, \tan \beta, m_{\tilde{\nu}}$ and $m_{\tilde{u}_{L}}=m_{\tilde{c}_{L}}$ and neutralino and chargino masses for scenario B for different values of $\phi_{\mu}$ and $\phi_{M_{1}} .\left|M_{1}\right|$ is fixed by the GUT-inspired relation $\left|M_{1}\right|=5 / 3 \tan ^{2} \theta_{W} M_{2}$ and the masses of the down-type sfermions by the $\mathrm{SU}(2)$ relation. All masses are given in $\mathrm{GeV}$.

\subsection{CP-odd asymmetry for $\tilde{\chi}_{1}^{+} \tilde{\chi}_{2}^{-}$production and $\tilde{\chi}_{1}^{+}$decay}

Now we consider the production process $e^{+} e^{-} \rightarrow \tilde{\chi}_{1}^{+} \tilde{\chi}_{2}^{-}$at $\sqrt{s}=500 \mathrm{GeV}$ with subsequent decays of the $\tilde{\chi}_{1}^{+}$. In this case $A_{\mathrm{CP}}\left(\mathcal{T}_{q}\right)$ is sensitive to the CP-violating couplings in the production and decay amplitudes (i.e. it is sensitive to both terms in (16)).

\subsubsection{Hadronic decay $\tilde{\chi}_{1}^{+} \rightarrow \tilde{\chi}_{1}^{0} \bar{s} c$}

In the case of hadronic decays, $\tilde{\chi}_{1}^{+} \rightarrow \tilde{\chi}_{1}^{0} \bar{s} c, c$-charge tagging is highly desirable because of the complicated cascade decays of the heavy chargino.

In Fig. 5 (a) we show the $\mathrm{CP}$ asymmetry $A_{\mathrm{CP}}\left(\mathcal{T}_{q}\right)$, Eq. (7), as a function of $\phi_{M_{1}}$ for scenario B given in Table 2, with $\phi_{\mu}=0$. The longitudinal beam polarization is $\left(P_{e^{-}}, P_{e^{+}}\right)=(-0.8,+0.6)((+0.8,-0.6))$. The asymmetry reaches its largest value of

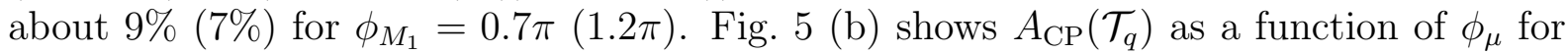
$\phi_{M_{1}}=0$. The largest value of the $\mathrm{CP}$ asymmetry is reached at $\phi_{\mu}=1.4 \pi(0.6 \pi)$. Note that the asymmetry can be large $(\sim 10 \%)$, even for values of $\phi_{\mu}$ close to $\pi$. As can be seen in Figs. [5 (a) and (b), it changes the sign for the two choices of beam polarizations.

In Fig. 66 (a) and (b) the contours of $A_{\mathrm{CP}}\left(\mathcal{T}_{q}\right)$, Eq. (7), are shown in the $M_{2^{-}}|\mu|$ plane for $\tan \beta=5, m_{\tilde{\nu}}=250 \mathrm{GeV}, m_{\tilde{c}}=500 \mathrm{GeV}, m_{\tilde{s}}=505.9 \mathrm{GeV},\left|M_{1}\right|=5 / 3 \tan ^{2} \theta_{W} M_{2}$, $\phi_{M_{1}}=0.5 \pi$ and $\phi_{\mu}=0$. Figs. 6 (c) and (d) show the corresponding contours for $\mathcal{N}_{\sigma}$, Eq. (18), for $\mathcal{L}_{\text {int }}=500 \mathrm{fb}^{-1}$ in the $M_{2^{-}}|\mu|$ plane. Also in this case the choice $\left(P_{e^{-}}, P_{e^{+}}\right)=$ $(-0.8,+0.6)$ enhances the statistical significance for a measurement of $A_{\mathrm{CP}}\left(\mathcal{T}_{q}\right)$.

\subsubsection{Leptonic decay $\tilde{\chi}_{1}^{+} \rightarrow \tilde{\chi}_{1}^{0} \ell^{+} \nu$}

In this section we analyse the CP asymmetry $A_{\mathrm{CP}}\left(\mathcal{T}_{\ell}\right)$, Eq. (77), based on the triple product correlation $\mathcal{T}_{\ell}=\vec{p}_{\ell^{+}} \cdot\left(\vec{p}_{e^{-}} \times \vec{p}_{\tilde{\chi}_{1}^{+}}\right)$. For the process $e^{+} e^{-} \rightarrow \tilde{\chi}_{1}^{+} \tilde{\chi}_{2}^{-}$the asymmetry $A_{\mathrm{CP}}\left(\mathcal{T}_{\ell}\right)$ is only sensitive to $\mathrm{CP}$-violating couplings in the production amplitude, which are involved in the first term of (16), because the CP sensitive couplings in the decay (c.f. Eqs. (66) -(69)) 
$A_{\mathrm{CP}}\left(\mathcal{T}_{q}\right)$ in $\%$

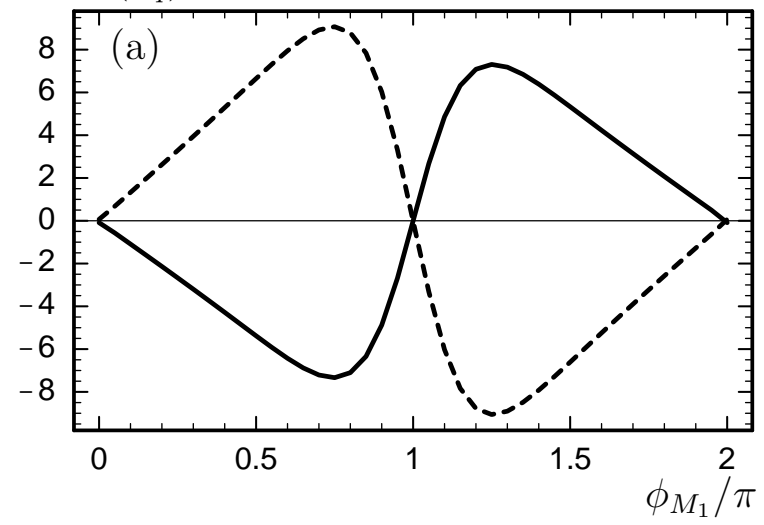

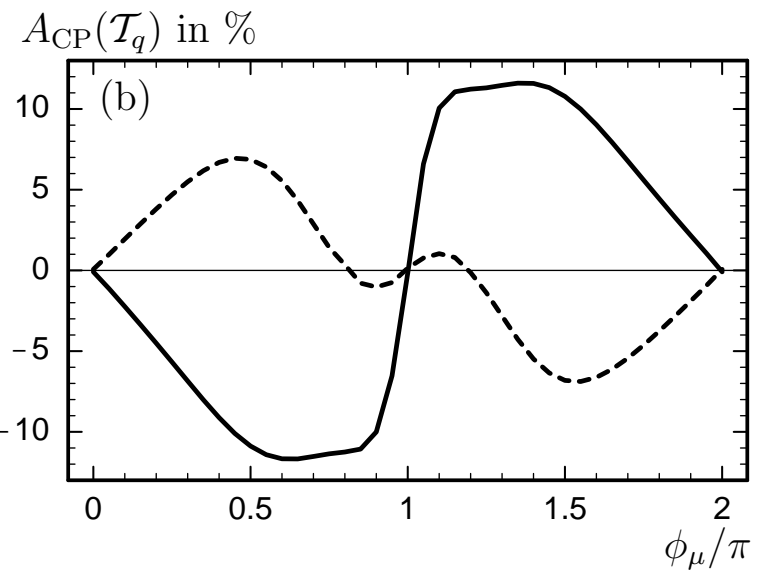

Figure 5: CP asymmetry $A_{\mathrm{CP}}\left(\mathcal{T}_{q}\right)$, Eq. (7), for $e^{+} e^{-} \rightarrow \tilde{\chi}_{1}^{+} \tilde{\chi}_{2}^{-}$with subsequent decay $\tilde{\chi}_{1}^{+} \rightarrow \tilde{\chi}_{1}^{0} \bar{s} c$ for the parameters given in Table 2 (a) with $\phi_{\mu}=0$ and (b) with $\phi_{M_{1}}=0$, for $\sqrt{s}=500 \mathrm{GeV}$ and for the beam polarizations $\left(P_{e^{-}}, P_{e^{+}}\right)=(-0.8,+0.6)$ (solid), $\left(P_{e^{-}}, P_{e^{+}}\right)=(+0.8,-0.6)$ (dashed).

do not contain the triple product $\mathcal{T}_{\ell}$. This means $A_{\mathrm{CP}}\left(\mathcal{T}_{\ell}\right)$ is proportional to $\sin \left(\phi_{\mu}\right)$ and therefore $A_{\mathrm{CP}}\left(\mathcal{T}_{\ell}\right) \equiv 0$ for $\phi_{\mu}=0, \pi, 2 \pi, \ldots$, independently of $\phi_{M_{1}}$. Hence, by measuring the $\mathrm{CP}$ asymmetries $A_{\mathrm{CP}}\left(\mathcal{T}_{\ell}\right)$ and $A_{\mathrm{CP}}\left(\mathcal{T}_{q}\right)$ one can separately study the influence of $\phi_{\mu}$ and $\phi_{M_{1}}$.

In Fig. 7 we show the contour lines of the CP-odd asymmetry $A_{\mathrm{CP}}\left(\mathcal{T}_{\ell}\right)$, Eq. (7), for scenario $\mathrm{C}$ of Table 3 in the $\phi_{M_{1}}-\phi_{\mu}$ plane. Fig. 7 illustrates that the asymmetry $A_{\mathrm{CP}}\left(\mathcal{T}_{\ell}\right)$ can be large for values of $\phi_{\mu}$ close to $\pi$. For instance, for $\phi_{M_{1}}=1.5 \pi$ and $\phi_{\mu}=0.9 \pi$ one obtains an asymmetry of about $23 \%$. However, the corresponding cross section is only about $0.16 \mathrm{fb}$.

In Fig. 8 (a) and (b), the $\mathrm{CP}$ asymmetry $A_{\mathrm{CP}}\left(\mathcal{T}_{\ell}\right)$, Eq. (7), and the number of standard deviations $\mathcal{N}_{\sigma}$, Eq. (18), are shown for $\mathcal{L}_{\text {int }}=500 \mathrm{fb}^{-1}$, respectively, in the $M_{2^{-}}|\mu|$ plane. The MSSM parameters $\operatorname{are} \tan \beta=5, m_{\tilde{\nu}}=250 \mathrm{GeV}, m_{\tilde{\ell}}=261.7 \mathrm{GeV}$, $\left|M_{1}\right|=5 / 3 \tan ^{2} \theta_{W} M_{2}, \phi_{M_{1}}=0$ and $\phi_{\mu}=0.5 \pi$. The asymmetry reaches its largest values of about $15 \%$ in gaugino-like scenarios. For example, for scenario $\mathrm{C}, A_{\mathrm{CP}}\left(\mathcal{T}_{\ell}\right)$ can be measured with a $5 \sigma$ significance.

In order to be able to measure $A_{C P}\left(\mathcal{T}_{\ell}\right)$, the production plane has to be reconstucted. Provided the masses of the particles are known, this could be accomplished depending on the decay pattern of $\tilde{\chi}_{2}^{-}$[13. For example, in the case of scenario $\mathrm{C}$ (fixing $\phi_{\mu}=\pi$ and $\left.\phi_{M_{1}}=0\right)$ the decays of $\tilde{\chi}_{2}^{-}$which can be used for the reconstruction of the production plane are (i) $\tilde{\chi}_{2}^{-} \rightarrow \tilde{\chi}_{2}^{0} W^{-}, \tilde{\chi}_{2}^{0} \rightarrow \tilde{\chi}_{1}^{0} q \bar{q}\left(\tilde{\chi}_{1}^{0} \ell \bar{\ell}\right), W^{-} \rightarrow q \bar{q}^{\prime}$, (ii) $\tilde{\chi}_{2}^{-} \rightarrow \tilde{\chi}_{1}^{-} Z^{0}, \tilde{\chi}_{1}^{-} \rightarrow \tilde{\chi}_{1}^{0} q \bar{q}^{\prime}$, $Z^{0} \rightarrow q \bar{q}(\ell \bar{\ell})$ and (iii) $\tilde{\chi}_{2}^{-} \rightarrow \tilde{\chi}_{1}^{-} h^{0}, \tilde{\chi}_{1}^{-} \rightarrow \tilde{\chi}_{1}^{0} q \bar{q}^{\prime}, h^{0} \rightarrow q \bar{q}(\ell \bar{\ell})$. The masses of the particles involved are given in Table 3, and we take $m_{h^{0}}=115 \mathrm{GeV}$. In the decay chains (i) ((ii), (iii)) we obtain two invariant mass constraints from the on-shell $\tilde{\chi}_{2}^{0}\left(\tilde{\chi}_{1}^{-}\right)$and the only invisible particle in the final states of the decay chains (i)-(iii) is $\tilde{\chi}_{1}^{0}$. In these cases the production plane can be reconstructed up to a twofold ambiguity. The branching ratios of the decay chains (i), (ii) and (iii) are $20 \%, 25 \%$ and $17 \%$, respectively. This means that in this case about $62 \%$ of the decays of $\tilde{\chi}_{2}^{-}$can be used for the reconstruction of 

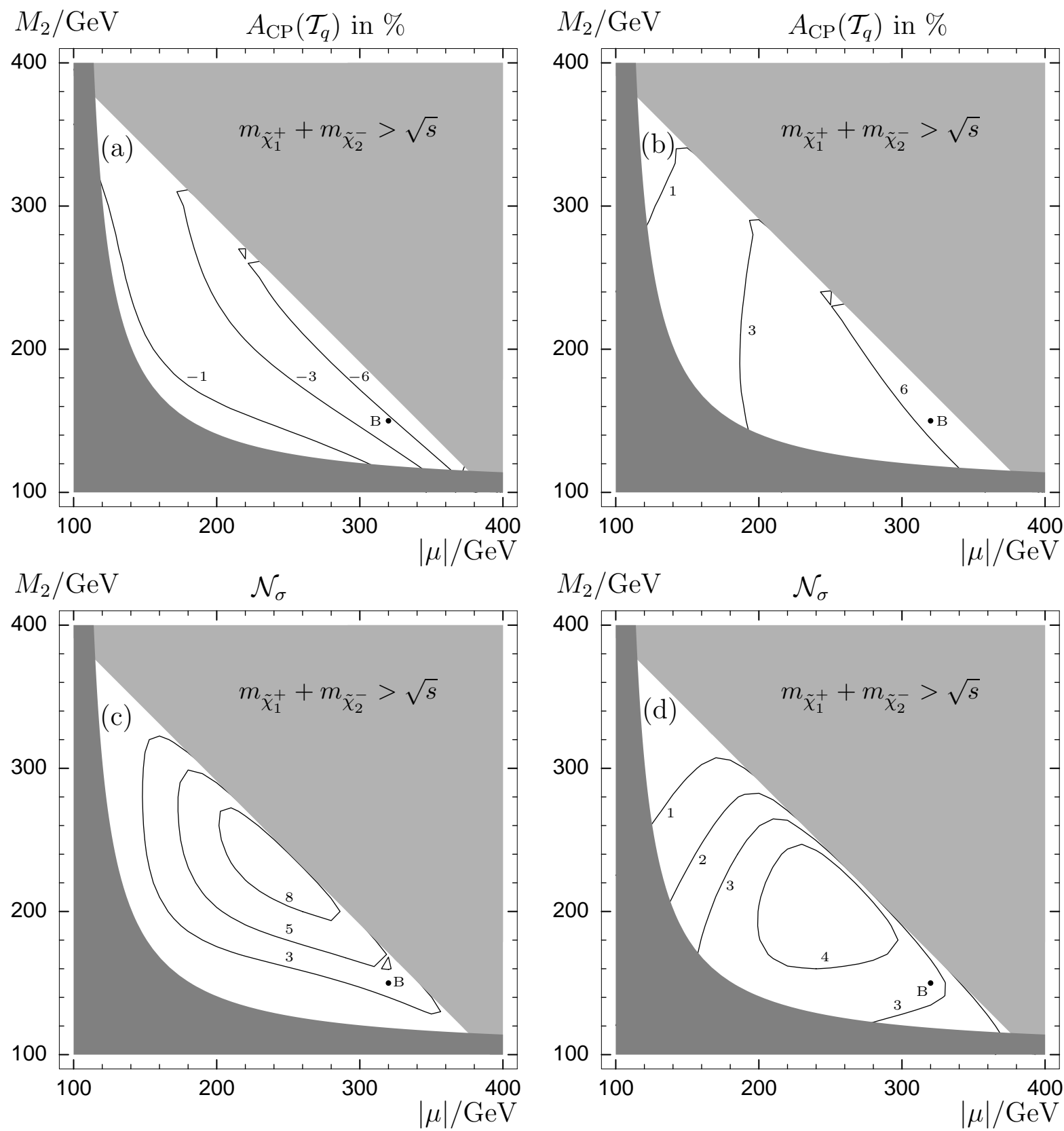

Figure 6: (a), (b) Contours of the CP asymmetry $A_{\mathrm{CP}}\left(\mathcal{T}_{q}\right)$, Eq. (7), in \% for $e^{+} e^{-} \rightarrow$ $\tilde{\chi}_{1}^{+} \tilde{\chi}_{2}^{-}$at $\sqrt{s}=500 \mathrm{GeV}$ with subsequent decay $\tilde{\chi}_{1}^{+} \rightarrow \tilde{\chi}_{1}^{0} \bar{s} c$ and (c), (d) contours of the number of standard deviations $\mathcal{N}_{\sigma}$, Eq. (18), for an integrated luminosity $\mathcal{L}_{\text {int }}=500 \mathrm{fb}^{-1}$, respectively. The parameters are $\tan \beta=5, m_{\tilde{\nu}}=250 \mathrm{GeV}, m_{\tilde{c}}=500 \mathrm{GeV}, m_{\tilde{s}}=$ $505.9 \mathrm{GeV},\left|M_{1}\right| / M_{2}=5 / 3 \tan ^{2} \theta_{W}, \phi_{M_{1}}=0.5 \pi$ and $\phi_{\mu}=0$. The beam polarizations are in $(\mathrm{a}),(\mathrm{c})\left(P_{e^{-}}, P_{e^{+}}\right)=(-0.8,+0.6)$ and in $(\mathrm{b}),(\mathrm{d})\left(P_{e^{-}}, P_{e^{+}}\right)=(+0.8,-0.6)$. The point marks the scenario B, defined in Table 2. In the dark-shaded area is $m_{\tilde{\chi}_{1}^{ \pm}}<103.5 \mathrm{GeV}$, excluded by LEP [28]. The light-shaded area is kinematically not accessible. 


\begin{tabular}{|c|c|c|c|c|c|c|c|c|c|}
\hline & $\phi_{\mu}$ & $\phi_{M_{1}}$ & $m_{\tilde{\chi}_{1}^{0}}$ & $m_{\tilde{\chi}_{2}^{0}}$ & $m_{\tilde{\chi}_{3}^{0}}$ & $m_{\tilde{\chi}_{4}^{0}}$ & $m_{\tilde{\chi}_{1}^{ \pm}}$ & $m_{\tilde{\chi}_{2}^{ \pm}}$ \\
\hline & & 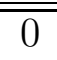 & 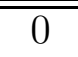 & $\overline{55.9}$ & 105.5 & 326.1 & 344.8 & 104.2 & 344.8 \\
\hline \multicolumn{2}{|c|}{ scenario C } & 0 & $\frac{\pi}{2}$ & 58.6 & 105.1 & 326.4 & 344.1 & 104.2 & 344.8 \\
\hline$M_{2}$ & 120 & 0 & $\pi$ & 61.4 & 104.5 & 326.8 & 343.5 & 104.2 & 344.8 \\
\hline$|\mu|$ & 320 & \multirow{3}{*}{$\begin{array}{l}\frac{\pi}{2} \\
\frac{\pi}{2} \\
\frac{\pi}{2} \\
\end{array}$} & & \multirow{3}{*}{$\begin{array}{l}59.1 \\
61.3 \\
58.7\end{array}$} & 112.7 & 327.5 & 340.6 & 112.5 & \multirow{3}{*}{$\begin{array}{l}342.2 \\
342.2 \\
342.2\end{array}$} \\
\hline $\tan \beta$ & 5 & & & & 112.5 & 328.0 & 339.7 & 112.5 & \\
\hline$m_{\tilde{\nu}}$ & 250 & & & & 112.8 & 328.1 & 340.1 & 112.5 & \\
\hline$m_{\tilde{u}_{L}}$ & 500 & $\frac{2}{\pi}$ & \multirow{2}{*}{$\begin{array}{l}0 \\
\frac{\pi}{2}\end{array}$} & \multirow{2}{*}{$\begin{array}{l}61.2 \\
59.1\end{array}$} & 120.2 & 331.9 & 333.3 & 120.5 & \multirow{2}{*}{$\begin{array}{l}339.4 \\
339.4\end{array}$} \\
\hline & & $\pi$ & & & 120.2 & 331.5 & 334.1 & 120.5 & \\
\hline & & $\pi$ & $\pi$ & 56.8 & 120.2 & 331.2 & 334.7 & 120.5 & 339.4 \\
\hline
\end{tabular}

Table 3: Input parameters $M_{2},|\mu|, \tan \beta, m_{\tilde{\nu}}$ and $m_{\tilde{u}_{L}}=m_{\tilde{c}_{L}}$ and neutralino and chargino masses for scenario $\mathrm{C}$ for different values of $\phi_{\mu}$ and $\phi_{M_{1}} .\left|M_{1}\right|$ is fixed by the GUT-inspired relation $\left|M_{1}\right|=5 / 3 \tan ^{2} \theta_{W} M_{2}$ and the masses of the down-type sfermions by the $\mathrm{SU}(2)$ relation. All masses are given in $\mathrm{GeV}$.

the production plane, which implies that the number of standard deviations $\mathcal{N}_{\sigma}$ shown in Fig. 8 (b) would have to be reduced accordingly. We note that in scenario $\mathrm{C}$ the decays $\tilde{\chi}_{2}^{-} \rightarrow \tilde{\tilde{\nu}} \ell^{-}$and $\tilde{\chi}_{2}^{-} \rightarrow \tilde{\ell}_{L}^{-} \bar{\nu}$ are suppressed and the decay $\tilde{\chi}_{2}^{-} \rightarrow \tilde{s} \bar{c}$ is kinematically not accessible. In the case that these decays contribute significantly, then it is again possible to reconstruct the production plane in the decays $\tilde{\chi}_{2}^{-} \rightarrow \overline{\tilde{\nu}} \ell^{-}$and $\tilde{\chi}_{2}^{-} \rightarrow \tilde{s} \bar{c}$.

In order to predict the significance more accurately, detailed Monte Carlo analysis including detector simulations and particle identification and reconstruction efficiencies would be required, which is, however, beyond the scope of the present work. For instance, a Monte Carlo analysis for a CP asymmetry in the production and decay of neutralinos with longitudinal beam polarization has been carried out in [11].

\section{Summary and conclusions}

We have analysed CP-sensitive observables in chargino production $e^{+} e^{-} \rightarrow \tilde{\chi}_{1}^{+} \tilde{\chi}_{1,2}^{-}$with subsequent hadronic and leptonic three-body decays $\tilde{\chi}_{1}^{+} \rightarrow \tilde{\chi}_{1}^{0} \bar{f}^{d} f^{u}\left(f^{d}=e, \mu, s\right.$ and $\left.f^{u}=\nu_{e}, \nu_{\mu}, c\right)$ at an $e^{+} e^{-}$linear collider with centre-of-mass energy $\sqrt{s}=500 \mathrm{GeV}$, integrated luminosity $\mathcal{L}_{\text {int }}=500 \mathrm{fb}^{-1}$ and longitudinally polarized beams. Our framework has been the MSSM with complex parameters. We have constructed CP-odd asymmetries with the help of triple product correlations between the momenta of the incoming and outgoing particles.

Considering the production process $e^{+} e^{-} \rightarrow \tilde{\chi}_{1}^{+} \tilde{\chi}_{1}^{-}$followed by the hadronic three-body decay $\tilde{\chi}_{1}^{+} \rightarrow \tilde{\chi}_{1}^{0} \bar{s} c$, we have defined the $\mathrm{CP}$ asymmetry $A_{\mathrm{CP}}\left(\mathcal{T}_{q}\right)$ that is based on the triple product $\mathcal{T}_{q}=\vec{p}_{\vec{s}} \cdot\left(\vec{p}_{c} \times \vec{p}_{e^{-}}\right)$. The asymmetry $A_{\mathrm{CP}}\left(\mathcal{T}_{q}\right)$ is sensitive to $\mathrm{CP}$ violation in the decay and depends on the phases $\phi_{\mu}$ and $\phi_{M_{1}}$ appearing in the chargino/neutralino system. We have shown that the measurability of the asymmetry $A_{\mathrm{CP}}\left(\mathcal{T}_{q}\right)$ can be significantly increased by a suitable choice of beam polarizations. Choosing $\left(P_{e^{-}}, P_{e^{+}}\right)=(-0.8,+0.6)$, $A_{\mathrm{CP}}\left(\mathcal{T}_{q}\right)$ can be probed at the $5 \sigma$ level in a large region of the MSSM parameter space. 


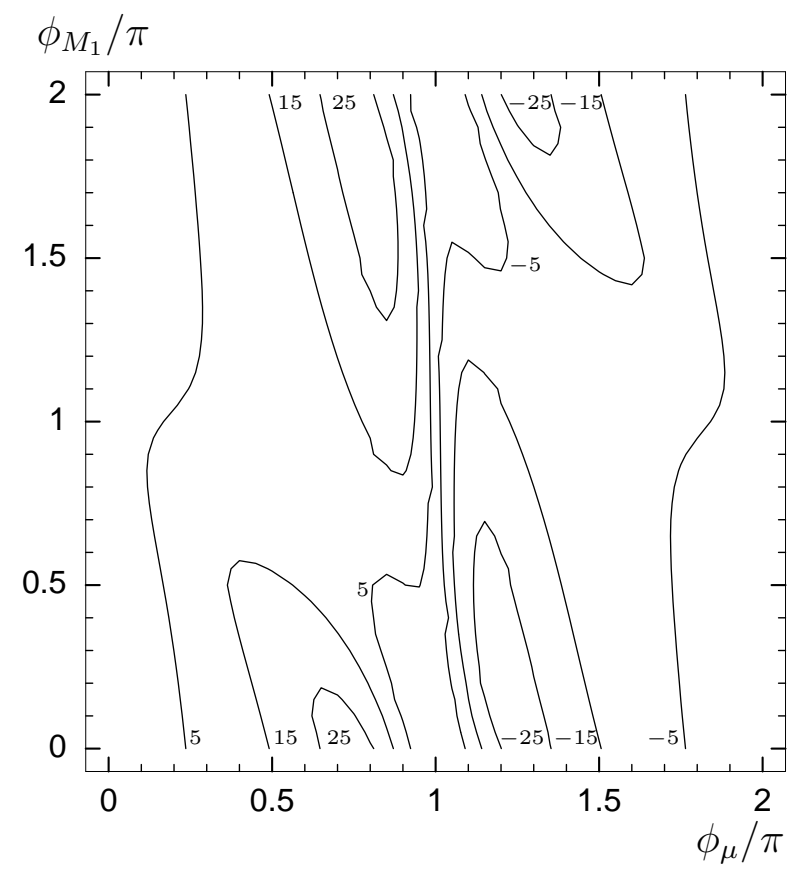

Figure 7: Contours of the $\mathrm{CP}$ asymmetry $A_{\mathrm{CP}}\left(\mathcal{T}_{\ell}\right)$, Eq. (7), in $\%$ for $e^{+} e^{-} \rightarrow \tilde{\chi}_{1}^{+} \tilde{\chi}_{2}^{-}$at $\sqrt{s}=500 \mathrm{GeV}$ with subsequent decay $\tilde{\chi}_{1}^{+} \rightarrow \tilde{\chi}_{1}^{0} \ell^{+} \nu$ for the parameters defined in Table 3 and $\left(P_{e^{-}}, P_{e^{+}}\right)=(-0.8,+0.6)$.

For the production process $e^{+} e^{-} \rightarrow \tilde{\chi}_{1}^{+} \tilde{\chi}_{2}^{-}$we have separately considered the hadronic three-body decay $\tilde{\chi}_{1}^{+} \rightarrow \tilde{\chi}_{1}^{0} \bar{s} c$ and the leptonic three-body decays $\tilde{\chi}_{1}^{+} \rightarrow \tilde{\chi}_{1}^{0} \ell^{+} \nu, \ell=e, \mu$. For the hadronic three-body decay we have studied again the CP asymmetry that is based on the triple product $\mathcal{T}_{q}$. In this case, the resulting $\mathrm{CP}$ asymmetry is sensitive to $\mathrm{CP}$ violation in production and decay. Also this asymmetry can be probed at the $5 \sigma$ level for MSSM parameters with appreciable gaugino-higgsino mixing. For the leptonic three-body decays, we have studied the asymmetry $A_{\mathrm{CP}}\left(\mathcal{T}_{\ell}\right)$ that is based on the triple product $\mathcal{T}_{\ell}=$ $\vec{p}_{\ell^{+}} \cdot\left(\vec{p}_{e^{-}} \times \vec{p}_{\tilde{\chi}_{i}^{+}}\right)$, which is sensitive to CP violation in the production only and hence to the phase $\phi_{\mu}$. We have found that the measurability of $A_{\mathrm{CP}}\left(\mathcal{T}_{\ell}\right)$ is somewhat decreased with respect to the previously considered asymmetries; however, in some regions of the MSSM parameter space it is accessible at the $3 \sigma$ level. As the two types of CP-odd asymmetries are sensitive to various combinations of the phases $\phi_{\mu}$ and $\phi_{M_{1}}$, their measurement will allow $\mathrm{CP}$ violation to be tested in the chargino/neutralino sector. Moreover, we have demonstrated that the CP-odd asymmetries studied in this paper can be large even for small CP-violating phases $\phi_{\mu}$ and $\phi_{M_{1}}$, which are favoured by the EDM constraints.

\section{Acknowledgements}

We thank O. Kittel, W. Majerotto and H.-U. Martyn for valuable discussions. This work is supported by the 'Fonds zur Förderung der wissenschaftlichen Forschung' (FWF) of Austria, project No. P18959-N16, and by the German Federal Ministry of Education and Research (BMBF) under contract number 05HT4WWA/2. The authors acknowledge 

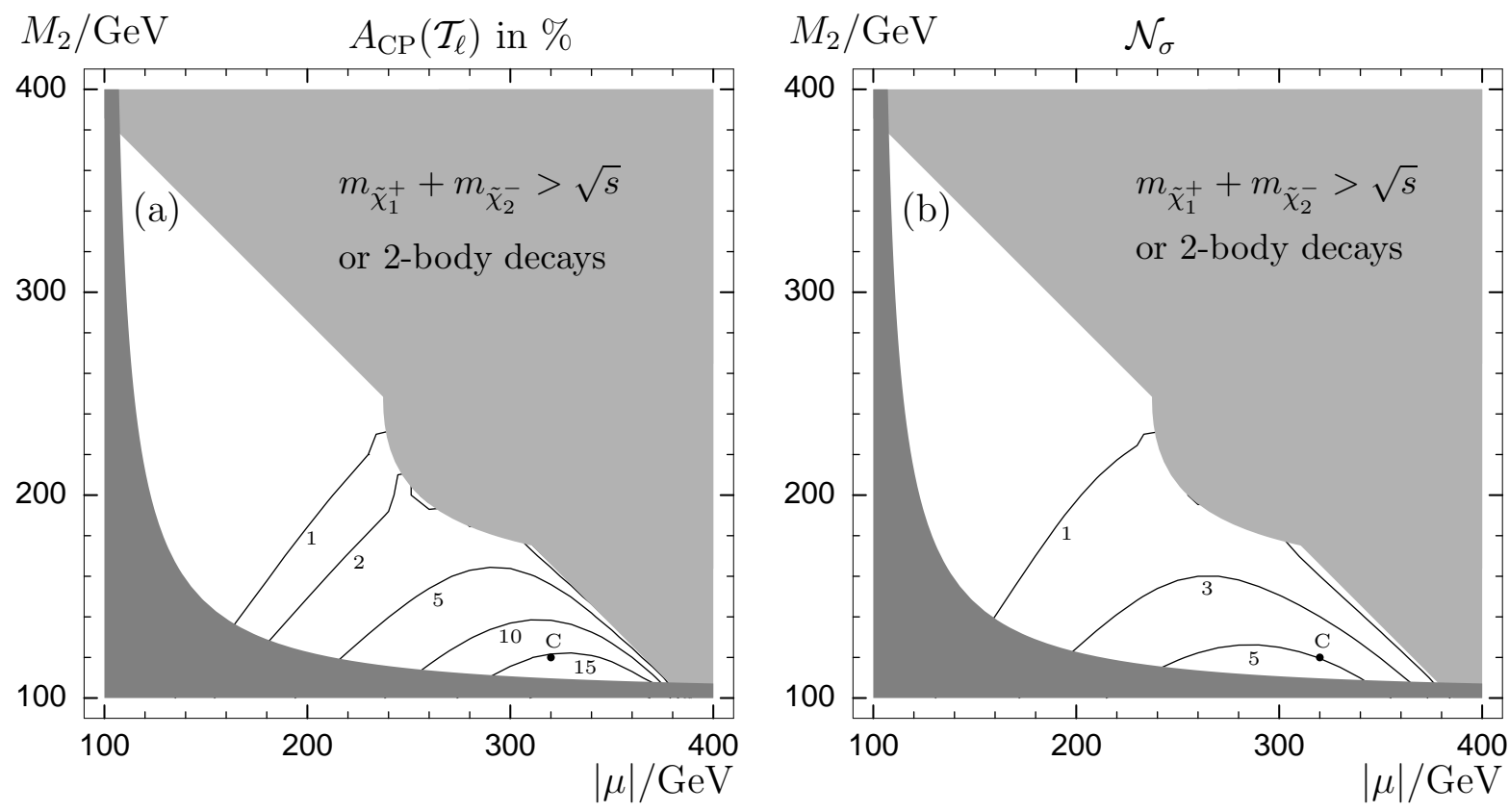

Figure 8: (a) Contours of the CP asymmetry $A_{\mathrm{CP}}\left(\mathcal{T}_{\ell}\right)$, Eq. (7), in $\%$ for $e^{+} e^{-} \rightarrow \tilde{\chi}_{1}^{+} \tilde{\chi}_{2}^{-}$ at $\sqrt{s}=500 \mathrm{GeV}$ with subsequent decay $\tilde{\chi}_{1}^{+} \rightarrow \tilde{\chi}_{1}^{0} \ell^{+} \nu$ and (b) contours of the number of standard deviations $\mathcal{N}_{\sigma}$, Eq. (18), for an integrated luminosity $\mathcal{L}_{\text {int }}=500 \mathrm{fb}^{-1}$, respectively. The parameters are $\tan \beta=5, m_{\tilde{\nu}}=250 \mathrm{GeV}, m_{\tilde{\ell}}=261.7 \mathrm{GeV}$, $\left|M_{1}\right| / M_{2}=5 / 3 \tan ^{2} \theta_{W}, \phi_{M_{1}}=0, \phi_{\mu}=0.5 \pi$ and the beam polarizations are $\left(P_{e^{-}}, P_{e^{+}}\right)=$ $(-0.8,+0.6)$. The point marks the scenario $\mathrm{C}$, defined in Table 3 , In the dark-shaded area is $m_{\tilde{\chi}_{1}^{ \pm}}<103.5 \mathrm{GeV}$, excluded by LEP [28]. The light-shaded area shows the region that either is not kinematically accessible or in which the three-body decay is strongly suppressed because $m_{\tilde{\chi}_{1}^{+}}>m_{W}+m_{\tilde{\chi}_{1}^{0}}$.

support from EU under the MRTN-CT-2006-035505 network programme.

\section{A Formalism}

The full expressions for the terms $\Sigma_{P}^{a}\left(\tilde{\chi}_{i}^{+}\right)$and $\Sigma_{D}^{a}\left(\tilde{\chi}_{i}^{+}\right)$are given in [24]. In the following we decompose $\Sigma_{P}^{a}\left(\tilde{\chi}_{i}^{+}\right)$and $\Sigma_{D}^{a}\left(\tilde{\chi}_{i}^{+}\right)$into T-odd and the T-even terms, which are needed in subsection 2.2.

\section{A.1 T-odd terms of production and T-even terms of decay}

In the definition of the momenta and polarization 4 -vectors we follow [12, 24]. $p_{i}, i=$ $1, \ldots, 7$, are the 4-momenta of the particles $e^{-}, e^{+}, \tilde{\chi}_{i}^{+}, \tilde{\chi}_{j}^{-}, \tilde{\chi}_{k}^{0}, f^{d}$ and $f^{u}$, respectively, see Figs. 1 and 2. It can be shown that all contributions to the T-odd terms $\Sigma_{P}^{a, \mathrm{O}}\left(\tilde{\chi}_{i}^{+}\right)$in Eq. (16) contain a factor

$$
f_{5}^{a}=i \cdot m_{j} \epsilon_{\mu \nu \rho \sigma} p_{2}^{\mu} p_{1}^{\nu} s^{a, \rho} p_{3}^{\sigma}
$$


which vanishes for longitudinal polarization $(a=3)$ and transverse polarization in the production plane $(a=1)$ so that we have only to include the spin terms for transverse polarization of the chargino $\tilde{\chi}_{i}^{+}$perpendicular to the production plane $(a=2)$ :

$$
\Sigma_{P}^{2, \mathrm{O}}\left(\tilde{\chi}_{i}^{+}\right)=\Sigma_{P}^{2, \mathrm{O}}(\gamma Z)+\Sigma_{P}^{2, \mathrm{O}}(\gamma \tilde{\nu})+\Sigma_{P}^{2, \mathrm{O}}(Z Z)+\Sigma_{P}^{2, \mathrm{O}}(Z \tilde{\nu})
$$

with

$$
\begin{aligned}
& \Sigma_{P}^{2, \mathrm{O}}(\gamma Z)=g^{4} \tan ^{2} \theta_{W} \operatorname{Re}\left\{\Delta(\gamma) \Delta^{*}(Z) \delta_{i j} c_{-}^{P}(\gamma Z)\left(O_{i j}^{\prime L *}-O_{i j}^{\prime R *}\right) f_{5}^{a=2}\right\}, \\
& \Sigma_{P}^{2, \mathrm{O}}(\gamma \tilde{\nu})=-\frac{g^{4}}{2} \sin ^{2} \theta_{W} \operatorname{Re}\left\{\Delta(\gamma) \Delta^{*}(\tilde{\nu}) \delta_{i j} c_{+}^{P}(\gamma \tilde{\nu}) V_{i 1}^{*} V_{j 1} f_{5}^{a=2}\right\}, \\
& \Sigma_{P}^{2, \mathrm{O}}(Z Z)=\frac{g^{4}}{2 \cos ^{4} \theta_{W}}|\Delta(Z)|^{2}\left[c_{-}^{P}(Z Z)\left(O_{i j}^{\prime R} O_{i j}^{\prime L *}-O_{i j}^{\prime L} O_{i j}^{\prime R *}\right) f_{5}^{a=2}\right], \\
& \Sigma_{P}^{2, \mathrm{O}}(Z \tilde{\nu})=-\frac{g^{4}}{2 \cos ^{2} \theta_{W}} \operatorname{Re}\left\{\Delta(Z) \Delta^{*}(\tilde{\nu}) c_{+}^{P}(Z \tilde{\nu}) V_{i 1}^{*} V_{j 1} O_{i j}^{\prime R} f_{5}^{a=2}\right\} .
\end{aligned}
$$

Here

$$
c_{ \pm}^{P}(\alpha \beta)= \pm c^{L}(\alpha) c^{L}(\beta)\left(1-P_{e^{-}}\right)\left(1+P_{e^{+}}\right)+c^{R}(\alpha) c^{R}(\beta)\left(1+P_{e^{-}}\right)\left(1-P_{e^{+}}\right)
$$

with

$$
\begin{aligned}
& c^{L}(\gamma)=1, \quad c^{L}(Z)=L_{e}, \quad c^{L}(\tilde{\nu})=1, \\
& c^{R}(\gamma)=1, \quad c^{R}(Z)=R_{e}, \quad c^{R}(\tilde{\nu})=0,
\end{aligned}
$$

and $P_{e^{-}}$and $P_{e^{+}}$is the degree of longitudinal polarization of the electron beam and positron beam, respectively. The propagators are $\Delta(\gamma)=i /\left(p_{1}+p_{2}\right)^{2}, \Delta(Z)=i /\left(\left(p_{1}+\right.\right.$ $\left.\left.p_{2}\right)^{2}-m_{Z}^{2}\right)$ and $\Delta(\tilde{\nu})=i /\left(\left(p_{1}-p_{4}\right)^{2}-m_{\tilde{\nu}}^{2}\right)$ and the couplings are given by

$$
\begin{aligned}
L_{f} & =T_{3 f}-e_{f} \sin ^{2} \theta_{W}, \quad R_{f}=-e_{f} \sin ^{2} \theta_{W}, \\
O_{i j}^{\prime L} & =-V_{i 1} V_{j 1}^{*}-\frac{1}{2} V_{i 2} V_{j 2}^{*}+\delta_{i j} \sin ^{2} \theta_{W}, \\
O_{i j}^{\prime} R & =-U_{i 1}^{*} U_{j 1}-\frac{1}{2} U_{i 2}^{*} U_{j 2}+\delta_{i j} \sin ^{2} \theta_{W},
\end{aligned}
$$

where $g$ is the weak coupling constant, $e_{f}$ and $T_{3 f}$ are the charge (in units of $e$ ) and the third component of the weak isospin of the fermion $f, \theta_{W}$ is the weak mixing angle. The unitary $(2 \times 2)$-matrices $U$ and $V$ diagonalize the complex chargino mass matrix, see for instance [4].

Note that $f_{5}^{a}$ is purely imaginary, so that, for example, $\Sigma_{P}^{2, \mathrm{O}}(\gamma Z)$, Eq. (21), is nonvanishing only if the couplings $O_{i j}^{\prime L, R}$ are complex and gives a CP-sensitive contribution to the asymmetry $A_{\mathrm{CP}}\left(\mathcal{T}_{q, \ell}\right)$. Analogous contributions come from the other terms in $\Sigma_{P}^{2, \mathrm{O}}$, Eqs. (22) $-(24)$. We have to multiply $\Sigma_{P}^{2, \mathrm{O}}$ in Eq. (16) by

$$
\begin{aligned}
\Sigma_{D}^{2, \mathrm{E}}\left(\tilde{\chi}_{i}^{+}\right)= & \Sigma_{D}^{2, \mathrm{E}}\left(W^{+} W^{+}\right)+\Sigma_{D}^{2, \mathrm{E}}\left(W^{+} \tilde{f}_{L}^{d}\right)+\Sigma_{D}^{2, \mathrm{E}}\left(W^{+} \tilde{f}_{L}^{u}\right) \\
& +\Sigma_{D}^{2, \mathrm{E}}\left(\tilde{f}_{L}^{d} \tilde{f}_{L}^{d}\right)+\Sigma_{D}^{2, \mathrm{E}}\left(\tilde{f}_{L}^{d} \tilde{f}_{L}^{u}\right)+\Sigma_{D}^{2, \mathrm{E}}\left(\tilde{f}_{L}^{u} \tilde{f}_{L}^{u}\right),
\end{aligned}
$$


with

$$
\begin{aligned}
\Sigma_{D}^{2, \mathrm{E}}\left(W^{+} W^{+}\right)= & 2 g^{4}|\Delta(W)|^{2}\left[2\left(\left|O_{k i}^{R}\right|^{2} g_{2}^{a=2}-\left|O_{k i}^{L}\right|^{2} g_{1}^{a=2}\right)\right. \\
& \left.-\left(O_{k i}^{L *} O_{k i}^{R}+O_{k i}^{L} O_{k i}^{R *}\right)\left(g_{4}^{a=2}-g_{3}^{a=2}\right)\right], \\
\Sigma_{D}^{2, \mathrm{E}}\left(W^{+} \tilde{f}_{L}^{d}\right)= & g^{4} \sqrt{2} \operatorname{Re}\left\{\Delta ( W ) \Delta ^ { * } ( \tilde { f } _ { L } ^ { d } ) 2 U _ { i 1 } ^ { * } f _ { f ^ { d } k } ^ { L } \left[2 O_{k i}^{R} g_{2}^{a=2}\right.\right. \\
& \left.\left.-O_{k i}^{L}\left(g_{4}^{a=2}-g_{3}^{a=2}\right)\right]\right\}, \\
\Sigma_{D}^{2, \mathrm{E}}\left(W^{+} \tilde{f}_{L}^{u}\right)= & g^{4} \sqrt{2} \operatorname{Re}\left\{\Delta ( W ) \Delta ^ { * } ( \tilde { f } _ { L } ^ { u } ) 2 V _ { i 1 } f _ { f ^ { u } k } ^ { L * } \left[2 O_{k i}^{L} g_{1}^{a=2}\right.\right. \\
& \left.\left.+O_{k i}^{R}\left(g_{4}^{a=2}-g_{3}^{a=2}\right)\right]\right\}, \\
\Sigma_{D}^{2, \mathrm{E}}\left(\tilde{f}_{L}^{d} \tilde{f}_{L}^{d}\right)= & 2 g^{4}\left|U_{i 1}\right|^{2}\left|f_{f^{d} k}^{L}\right|^{2}\left|\Delta\left(\tilde{f}_{L}^{d}\right)\right|^{2} g_{2}^{a=2}, \\
\Sigma_{D}^{2, \mathrm{E}}\left(\tilde{f}_{L}^{d} \tilde{f}_{L}^{u}\right)= & 2 g^{4} \operatorname{Re}\left\{\Delta\left(\tilde{f}_{L}^{d}\right) \Delta^{*}\left(\tilde{f}_{L}^{u}\right) U_{i 1} f_{f^{d} k}^{L *} V_{i 1} f_{f^{u} k}^{L *}\left(g_{4}^{a=2}-g_{3}^{a=2}\right)\right\}, \\
\Sigma_{D}^{2, \mathrm{E}}\left(\tilde{f}_{L}^{u} \tilde{f}_{L}^{u}\right)= & -2 g^{4}\left|V_{i 1}\right|^{2}\left|f_{f^{u} k}^{L}\right|^{2}\left|\Delta\left(\tilde{f}_{L}^{u}\right)\right|^{2} g_{1}^{a=2},
\end{aligned}
$$

where

$$
\begin{aligned}
& g_{1}^{a=2}=m_{i}\left(p_{5} p_{7}\right)\left(p_{6} s^{a=2}\right), \\
& g_{2}^{a=2}=m_{i}\left(p_{5} p_{6}\right)\left(p_{7} s^{a=2}\right), \\
& g_{3}^{a=2}=m_{k}\left(p_{3} p_{7}\right)\left(p_{6} s^{a=2}\right), \\
& g_{4}^{a=2}=m_{k}\left(p_{3} p_{6}\right)\left(p_{7} s^{a=2}\right) .
\end{aligned}
$$

The propagators are $\Delta(W)=i /\left(\left(p_{3}-p_{5}\right)^{2}-m_{W}^{2}\right), \Delta\left(\tilde{f}_{L}^{u}\right)=i /\left(\left(p_{3}-p_{6}\right)^{2}-m_{\tilde{f}_{L}^{u}}^{2}\right)$ and $\Delta\left(\tilde{f}_{L}^{d}\right)=i /\left(\left(p_{3}-p_{7}\right)^{2}-m_{\tilde{f}_{L}^{d}}^{2}\right)$ and the couplings are given by

$$
\begin{aligned}
f_{f k}^{L} & =-\sqrt{2}\left[\frac{1}{\cos \theta_{W}}\left(T_{3 f}-e_{f} \sin ^{2} \theta_{W}\right) N_{k 2}+e_{f} \sin \theta_{W} N_{k 1}\right] \\
O_{k i}^{L} & =-1 / \sqrt{2}\left(\cos \beta N_{k 4}-\sin \beta N_{k 3}\right) V_{i 2}^{*}+\left(\sin \theta_{W} N_{k 1}+\cos \theta_{W} N_{k 2}\right) V_{i 1}^{*} \\
O_{k i}^{R} & =+1 / \sqrt{2}\left(\sin \beta N_{k 4}^{*}+\cos \beta N_{k 3}^{*}\right) U_{i 2}+\left(\sin \theta_{W} N_{k 1}^{*}+\cos \theta_{W} N_{k 2}^{*}\right) U_{i 1},
\end{aligned}
$$

where $\tan \beta=v_{2} / v_{1}$ is the ratio of the vacuum expectation values of the Higgs fields and the unitary $(4 \times 4)$-matrix $N$ diagonalizes the complex symmetric neutralino mass matrix which is given in the basis $\left(\tilde{\gamma}, \tilde{Z}, \tilde{H}_{a}^{0}, \tilde{H}_{b}^{0}\right)$ [30].

The kinematic functions $g_{1}^{a}, g_{2}^{a}, g_{3}^{a}, g_{4}^{a}, a=2$ are real. When multiplied by the purely imaginary $f_{5}^{a=2}$, Eq. (19), this leads to triple products sensitive to the CP phases of the couplings $O_{i j}^{\prime L, R}$ in the production process, which in the laboratory system read:

$$
\begin{aligned}
& g_{1}^{a=2} \cdot f_{5}^{a=2}=i 2 E_{b} m_{i} m_{j}\left(p_{5} p_{7}\right) \vec{p}_{6}\left(\vec{p}_{1} \times \vec{p}_{3}\right), \\
& g_{2}^{a=2} \cdot f_{5}^{a=2}=i 2 E_{b} m_{i} m_{j}\left(p_{5} p_{6}\right) \vec{p}_{7}\left(\vec{p}_{1} \times \vec{p}_{3}\right), \\
& g_{3}^{a=2} \cdot f_{5}^{a=2}=i 2 E_{b} m_{j} m_{k}\left(p_{3} p_{7}\right) \vec{p}_{6}\left(\vec{p}_{1} \times \vec{p}_{3}\right), \\
& g_{4}^{a=2} \cdot f_{5}^{a=2}=i 2 E_{b} m_{j} m_{k}\left(p_{3} p_{6}\right) \vec{p}_{7}\left(\vec{p}_{1} \times \vec{p}_{3}\right) .
\end{aligned}
$$

As outlined above, these expressions will be multiplied in Eqs. (21)-(24) by the factors $i \cdot \operatorname{Im}\left\{\left(O_{i j}^{\prime L *}-O_{i j}^{\prime R *}\right)\right\}$ etc., and contribute to the first term of Eq. (16) and, hence, to the numerator of the asymmetry $A_{T}$, Eq. (13). 


\section{A.2 T-odd terms of decay and T-even terms of production}

The factor

$$
\Sigma_{D}^{a, \mathrm{O}}\left(\tilde{\chi}_{i}^{+}\right)=\Sigma_{D}^{a, \mathrm{O}}\left(W^{+} W^{+}\right)+\Sigma_{D}^{a, \mathrm{O}}\left(W^{+} \tilde{f}_{L}^{d}\right)+\Sigma_{D}^{a, \mathrm{O}}\left(W^{+} \tilde{f}_{L}^{u}\right)+\Sigma_{D}^{a, \mathrm{O}}\left(\tilde{f}_{L}^{d} \tilde{f}_{L}^{u}\right)
$$

in the second term in Eq. (16) with

$$
\begin{aligned}
\Sigma_{D}^{a, \mathrm{O}}\left(W^{+} W^{+}\right) & =2 g^{4}|\Delta(W)|^{2} \operatorname{Re}\left\{\left(O_{k i}^{L *} O_{k i}^{R}-O_{k i}^{L} O_{k i}^{R *}\right) g_{5}^{a}\right\}, \\
\Sigma_{D}^{a, \mathrm{O}}\left(W^{+} \tilde{f}_{L}^{d}\right) & =-\sqrt{2} g^{4} \operatorname{Re}\left\{\Delta(W) \Delta^{*}\left(\tilde{f}_{L}^{d}\right) 2 U_{i 1}^{*} f_{f^{d} k}^{L} O_{k i}^{L} g_{5}^{a}\right\}, \\
\Sigma_{D}^{a, \mathrm{O}}\left(W^{+} \tilde{f}_{L}^{u}\right) & =-\sqrt{2} g^{4} \operatorname{Re}\left\{\Delta(W) \Delta^{*}\left(\tilde{f}_{L}^{u}\right) 2 V_{i 1} f_{f^{u} k}^{L *} O_{k i}^{R} g_{5}^{a}\right\}, \\
\Sigma_{D}^{a, \mathrm{O}}\left(\tilde{f}_{L}^{d} \tilde{f}_{L}^{u}\right) & =-2 \operatorname{Re}\left\{\Delta\left(\tilde{f}_{L}^{d}\right) \Delta^{*}\left(\tilde{f}_{L}^{u}\right) U_{i 1} f_{f^{d} k}^{L *} V_{i 1} f_{f^{u} k}^{L *} g_{5}^{a}\right\}
\end{aligned}
$$

is sensitive to $\mathrm{CP}$ violation in the decay of the chargino $\tilde{\chi}_{i}^{+}$[12, 24] due to the purely imaginary kinematic factor

$$
g_{5}^{a}=i \cdot m_{k} \epsilon_{\mu \nu \rho \sigma} s^{a \mu} p_{3}^{\nu} p_{7}^{\rho} p_{6}^{\sigma}
$$

For example in Eq. (50) it is multiplied by the factor $i \cdot \operatorname{Im}\left\{\left(O_{k i}^{L *} O_{k i}^{R}-O_{k i}^{L} O_{k i}^{R *}\right)\right\}$, which depends on the phases $\phi_{\mu}$ and $\phi_{M_{1}}$ and contributes to the CP asymmetry $A_{\mathrm{CP}}$, Eq. (7). Analogous contributions follow from Eqs. (51)-(53).

The T-even contributions from the production process in Eq. (16) are

$$
\begin{aligned}
\Sigma_{P}^{a, \mathrm{E}}\left(\tilde{\chi}_{i}^{+}\right)= & \Sigma_{P}^{a, \mathrm{E}}(\gamma \gamma)+\Sigma_{P}^{a, \mathrm{E}}(\gamma Z)+\Sigma_{P}^{a, \mathrm{E}}(\gamma \tilde{\nu}) \\
& +\Sigma_{P}^{a, \mathrm{E}}(Z Z)+\Sigma_{P}^{a, \mathrm{E}}(Z \tilde{\nu})+\Sigma_{P}^{a, \mathrm{E}}(\tilde{\nu} \tilde{\nu}),
\end{aligned}
$$

with

$$
\begin{aligned}
\Sigma_{P}^{a, \mathrm{E}}(\gamma \gamma)= & g^{4} \sin ^{2} \theta_{W}|\Delta(\gamma)|^{2} c_{-}^{P}(\gamma \gamma) \delta_{i j}\left(-f_{1}^{a}+f_{2}^{a}+f_{4}^{a}-f_{3}^{a}\right) \\
\Sigma_{P}^{a, \mathrm{E}}(\gamma Z)= & g^{4} \tan ^{2} \theta_{W} \operatorname{Re}\left\{\Delta ( \gamma ) \Delta ^ { * } ( Z ) \delta _ { i j } \left[c_{+}^{P}(\gamma Z)\left(O_{i j}^{\prime R *}-O_{i j}^{\prime L *}\right)\left(f_{1}^{a}+f_{2}^{a}\right)\right.\right. \\
& \left.\left.+c_{-}^{P}(\gamma Z)\left(O_{i j}^{\prime R *}+O_{i j}^{\prime L *}\right)\left(-f_{1}^{a}+f_{2}^{a}+f_{4}^{a}-f_{3}^{a}\right)\right]\right\} \\
\Sigma_{P}^{a, \mathrm{E}}(\gamma \tilde{\nu})= & -\frac{g^{4}}{2} \sin ^{2} \theta_{W} \operatorname{Re}\left\{\Delta(\gamma) \Delta^{*}(\tilde{\nu}) \delta_{i j} c_{+}^{P}(\gamma \tilde{\nu}) V_{i 1}^{*} V_{j 1}\left(2 f_{2}^{a}+f_{4}^{a}-f_{3}^{a}\right)\right\} \\
\Sigma_{P}^{a, \mathrm{E}}(Z Z)= & \frac{g^{4}}{2 \cos ^{4} \theta_{W}}|\Delta(Z)|^{2}\left[c_{+}^{P}(Z Z)\left(\left|O_{i j}^{\prime R}\right|^{2}-\left|O_{i j}^{\prime L}\right|^{2}\right)\left(f_{1}^{a}+f_{2}^{a}\right)\right. \\
& +c_{-}^{P}(Z Z)\left(\left(O_{i j}^{\prime} O_{i j}^{\prime R *}+O_{i j}^{\prime} O_{i j}^{\prime}{ }^{L *}\right)\left(f_{4}^{a}-f_{3}^{a}\right)\right. \\
& \left.\left.+\left(\left|O_{i j}^{\prime}\right|^{2}+\left|O_{i j}^{\prime L}\right|^{2}\right)\left(-f_{1}^{a}+f_{2}^{a}\right)\right)\right] \\
\Sigma_{P}^{a, \mathrm{E}}(Z \tilde{\nu})= & -\frac{g^{4}}{2 \cos ^{2} \theta_{W}} \operatorname{Re}\left\{\Delta(Z) \Delta^{*}(\tilde{\nu}) c_{+}^{P}(Z \tilde{\nu}) V_{i 1}^{*} V_{j 1}\left(2 O_{i j}^{L} f_{2}^{a}+O_{i j}^{\prime R}\left(f_{4}^{a}-f_{3}^{a}\right)\right)\right\}, \\
\Sigma_{P}^{a, \mathrm{E}}(\tilde{\nu} \tilde{\nu})= & -\frac{g^{4}}{4}\left|V_{i 1}\right|^{2}\left|V_{j 1}\right|^{2}|\Delta(\tilde{\nu})|^{2} c_{+}^{P}(\tilde{\nu} \tilde{\nu}) f_{2}^{a},
\end{aligned}
$$


where

$$
\begin{aligned}
& f_{1}^{a}=m_{i}\left(p_{2} p_{4}\right)\left(p_{1} s^{a}\right) \\
& f_{2}^{a}=m_{i}\left(p_{1} p_{4}\right)\left(p_{2} s^{a}\right), \\
& f_{3}^{a}=m_{j}\left(p_{2} p_{3}\right)\left(p_{1} s^{a}\right), \\
& f_{4}^{a}=m_{j}\left(p_{1} p_{3}\right)\left(p_{2} s^{a}\right) .
\end{aligned}
$$

Since $s^{a}\left(\tilde{\chi}_{i}^{+}\right)$for $a=2$ is perpendicular to the production plane, $\Sigma_{P}^{2, \mathrm{E}}\left(\tilde{\chi}_{i}^{+}\right)$vanishes, so that in $A_{\mathrm{CP}}$ only the contributions of the longitudinal polarization $(a=3)$ and of the transverse polarization in the production plane $(a=1)$ have to be taken into account.

Finally, the triple products sensitive to the $\mathrm{CP}$ phases in the chargino decay in the laboratory system read

$$
\begin{aligned}
\sum_{a=1,3} f_{1}^{a} \cdot g_{5}^{a}=i m_{i} m_{k}\left(p_{2} p_{4}\right)\{ & -E_{b} \vec{p}_{5}\left(\vec{p}_{7} \times \vec{p}_{6}\right)-E_{7} \vec{p}_{5}\left(\vec{p}_{6} \times \vec{p}_{1}\right) \\
& \left.+E_{6} \vec{p}_{5}\left(\vec{p}_{7} \times \vec{p}_{1}\right)+E_{5} \vec{p}_{1}\left(\vec{p}_{7} \times \vec{p}_{6}\right)\right\}, \\
\sum_{a=1,3} f_{2}^{a} \cdot g_{5}^{a}=i m_{i} m_{k}\left(p_{1} p_{4}\right)\{ & -E_{b} \vec{p}_{5}\left(\vec{p}_{7} \times \vec{p}_{6}\right)+E_{7} \vec{p}_{5}\left(\vec{p}_{6} \times \vec{p}_{1}\right) \\
& \left.-E_{6} \vec{p}_{5}\left(\vec{p}_{7} \times \vec{p}_{1}\right)-E_{5} \vec{p}_{1}\left(\vec{p}_{7} \times \vec{p}_{6}\right)\right\}, \\
\sum_{a=1,3} f_{3}^{a} \cdot g_{5}^{a}=i m_{j} m_{k}\left(p_{2} p_{3}\right)\{ & -E_{b} \vec{p}_{5}\left(\vec{p}_{7} \times \vec{p}_{6}\right)-E_{7} \vec{p}_{5}\left(\vec{p}_{6} \times \vec{p}_{1}\right) \\
& \left.+E_{6} \vec{p}_{5}\left(\vec{p}_{7} \times \vec{p}_{1}\right)+E_{5} \vec{p}_{1}\left(\vec{p}_{7} \times \vec{p}_{6}\right)\right\}, \\
\sum_{a=1,3} f_{4}^{a} \cdot g_{5}^{a}=i m_{j} m_{k}\left(p_{1} p_{3}\right)\{ & -E_{b} \vec{p}_{5}\left(\vec{p}_{7} \times \vec{p}_{6}\right)+E_{7} \vec{p}_{5}\left(\vec{p}_{6} \times \vec{p}_{1}\right) \\
& \left.-E_{6} \vec{p}_{5}\left(\vec{p}_{7} \times \vec{p}_{1}\right)-E_{5} \vec{p}_{1}\left(\vec{p}_{7} \times \vec{p}_{6}\right)\right\} .
\end{aligned}
$$

\section{References}

[1] H. P. Nilles, Phys. Rep. 110 (1984) 1;

H. E. Haber and G. L. Kane, Phys. Rep. 117 (1985) 75;

R. Barbieri, Riv. Nuovo Cim. 11 (1988) 1;

M. Drees, R. Godbole and P. Roy, "Theory and phenomenology of sparticles: An account of four-dimensional $N=1$ supersymmetry in high energy physics," (World Scientific, Hackensack, USA, 2004) p. 555;

D. J. H. Chung, L. L. Everett, G. L. Kane, S. F. King, J. D. Lykken and L. T. Wang, Phys. Rept. 407 (2005) 1 hep-ph/0312378].

[2] TESLA Technical Design Report, Part: III Physics at an $e^{+} e^{-}$Linear Collider, eds. R.-D. Heuer, D. Miller, F. Richard and P. Zerwas, DESY 2001-011, hep-ph/0106315; T. Abe et al. [American Linear Collider Working Group Collaboration], "Linear collider physics resource book for Snowmass 2001. 2: Higgs and supersymmetry studies," in Proc. of the APS/DPF/DPB Summer Study on the Future of Particle Physics 
(Snowmass 2001), ed. N. Graf, hep-ex/0106056;

K. Abe et al., JLC Roadmap Report, presented at the ACFA LC Symposium, Tsukuba, Japan 2003, http://lcdev.kek.jp/RMdraft/

[3] J. A. Aguilar-Saavedra et al., Eur. Phys. J. C 46 (2006) 43 hep-ph/0511344.

[4] S. Y. Choi, A. Djouadi, H. K. Dreiner, J. Kalinowski and P. M. Zerwas, Eur. Phys. J. C 7 (1999) 123 hep-ph/9806279];

S. Y. Choi, A. Djouadi, H. S. Song and P. M. Zerwas, Eur. Phys. J. C 8 (1999) 669 [hep-ph/9812236];

S. Y. Choi, A. Djouadi, M. Guchait, J. Kalinowski, H. S. Song and P. M. Zerwas, Eur. Phys. J. C 14 (2000) 535 hep-ph/0002033;

S. Y. Choi, M. Guchait, J. Kalinowski and P. M. Zerwas, Phys. Lett. B 479 (2000) 235 hep-ph/0001175.

[5] V. D. Barger, T. Han, T. J. Li and T. Plehn, Phys. Lett. B 475 (2000) 342 hep-ph/9907425];

J. L. Kneur and G. Moultaka, parameters Phys. Rev. D 61 (2000) 095003 hep-ph/9907360];

G. J. Gounaris, C. Le Mouel and P. I. Porfyriadis, Phys. Rev. D 65 (2002) 035002 hep-ph/0107249;

S. Y. Choi, J. Kalinowski, G. A. Moortgat-Pick and P. M. Zerwas, Eur. Phys. J. C 22 (2001) 563 [Addendum-ibid. C 23 (2002) 769] [hep-ph/0108117];

S. Y. Choi, J. Kalinowski, G. A. Moortgat-Pick and P. M. Zerwas, [hep-ph/0202039];

G. J. Gounaris and C. Le Mouel, Phys. Rev. D 66 (2002) 055007 [hep-ph/0204152].

[6] For a review see, for instance, T. Ibrahim and P. Nath, hep-ph/0107325 and hep-ph/0210251.

[7] T. Ibrahim and P. Nath, Phys. Lett. B 418 (1998) 98 hep-ph/9707409]; Phys. Rev. D 57 (1998) 478 [Erratum, ibid. D 58 (1998) 019901, D 60 (1999) 079903, and 119901] hep-ph/9708456]; Phys. Rev. D 58 (1998) 111301 [Erratum, ibid. D 60 (1999) 099902] [hep-ph/9807501]; Phys. Rev. D 61 (2000) 093004 [hep-ph/9910553];

M. Brhlik, G. J. Good and G. L. Kane, Phys. Rev. D 59 (1999) 115004 [hep-ph/9810457];

M. Brhlik, L. L. Everett, G. L. Kane and J. Lykken, Phys. Rev. Lett. 83 (1999) 2124 [hep-ph/9905215]; Phys. Rev. D 62 (2000) 035005 [hep-ph/9908326];

A. Bartl, T. Gajdosik, W. Porod, P. Stockinger and H. Stremnitzer, Phys. Rev. D 60 (1999) 073003 hep-ph/9903402;

A. Bartl, T. Gajdosik, E. Lunghi, A. Masiero, W. Porod, H. Stremnitzer and O. Vives, Phys. Rev. D 64 (2001) 076009 [hep-ph/0103324];

S. Abel, S. Khalil and O. Lebedev, Nucl. Phys. B 606 (2001) 151 [hep-ph/0103320].

[8] A. Bartl, W. Majerotto, W. Porod and D. Wyler, Phys. Rev. D 68 (2003) 053005 [hep-ph/0306050].

[9] S. Yaser Ayazi and Y. Farzan, Phys. Rev. D 74 (2006) 055008 hep-ph/0605272. 
[10] Y. Kizukuri and N. Oshimo, Phys. Lett. B 249 (1990) 449;

S. Y. Choi, H. S. Song and W. Y. Song, Phys. Rev. D 61 (2000) 075004 hep-ph/9907474;

V. D. Barger, T. Falk, T. Han, J. Jiang, T. Li and T. Plehn, Phys. Rev. D 64 (2001) 056007 hep-ph/0101106];

A. Bartl, H. Fraas, O. Kittel and W. Majerotto, Phys. Rev. D 69 (2004) 035007 hep-ph/0308141;

A. Bartl, T. Kernreiter and O. Kittel, Phys. Lett. B 578 (2004) 341 [hep-ph/0309340];

S. Y. Choi, M. Drees, B. Gaissmaier and J. Song, Phys. Rev. D 69 (2004) 035008 [hep-ph/0310284];

A. Bartl, H. Fraas, O. Kittel and W. Majerotto, Eur. Phys. J. C 36 (2004) 233 [hep-ph/0402016];

S. Y. Choi, M. Drees and B. Gaissmaier, Phys. Rev. D 70 (2004) 014010 hep-ph/0403054;

S. Y. Choi, B. C. Chung, J. Kalinowski, Y. G. Kim and K. Rolbiecki, Eur. Phys. J. C 46 (2006) 511 hep-ph/0504122].

[11] J. A. Aguilar-Saavedra, Nucl. Phys. B 697, 207 (2004) hep-ph/0404104.

[12] A. Bartl, H. Fraas, S. Hesselbach, K. Hohenwarter-Sodek and G. A. Moortgat-Pick, JHEP 0408 (2004) 038 [hep-ph/0406190];

S. Hesselbach, Acta Phys. Polon. B 35 (2004) 2739 [hep-ph/0410174].

[13] A. Bartl, H. Fraas, S. Hesselbach, K. Hohenwarter-Sodek, T. Kernreiter and G. A. Moortgat-Pick, JHEP 0601 (2006) 170 [hep-ph/0510029];

S. Y. Choi, M. Drees and J. Song, JHEP 0609 (2006) 064 [hep-ph/0602131].

[14] W. M. Yang and D. S. Du, Phys. Rev. D 67 (2003) 055004 hep-ph/0211453;

H. Eberl, T. Gajdosik, W. Majerotto and B. Schrausser, Phys. Lett. B 618 (2005) 171 hep-ph/0502112.

[15] J. A. Aguilar-Saavedra, Nucl. Phys. B 717 (2005) 119 hep-ph/0410068.

[16] O. Kittel, A. Bartl, H. Fraas and W. Majerotto, Phys. Rev. D 70 (2004) 115005 hep-ph/0410054.

[17] A. Bartl, H. Fraas, O. Kittel and W. Majerotto, Phys. Lett. B 598 (2004) 76 hep-ph/0406309.

[18] A. Bartl, K. Hohenwarter-Sodek, T. Kernreiter and H. Rud, Eur. Phys. J. C 36 (2004) 515 [hep-ph/0403265].

[19] G. Alexander et al., TESLA Technical Design Report, Part IV, 'A detector for TESLA', eds. T. Behnke, S. Bertolucci, R.-D. Heuer, R. Settles, DESY 2001-011.

[20] S. M. Xella Hansen, M. Wing, D. J. Jackson, N. De Groot and C. J. S. Damerell, LC-PHSM-2003-061; 
S. M. Xella Hansen [Linear Collider Flavour Identification Collaboration], Nucl. Instrum. Meth. A 501, 106 (2003);

S. Hillert and C. J. S. Damerell, eConf C0508141 (2005) ALCPG1403.

[21] C. J. S. Damerell and D. J. Jackson, eConf C960625 (1996) DET078;

R. Hawkings, LC-PHSM-2000-021;

S. M. Xella Hansen, D. J. Jackson, R. Hawkings and C. J. S. Damerell, LC-PHSM2001-024.

[22] C. J. S. Damerell, private communication.

[23] K. Abe et al. [SLD Collaboration], Phys. Rev. Lett. 88 (2002) 151801 hep-ex/0111035.

[24] G. A. Moortgat-Pick, H. Fraas, A. Bartl and W. Majerotto, Eur. Phys. J. C 7 (1999) 113 hep-ph/9804306.

[25] H. E. Haber, Proceedings of the 21st SLAC Summer Institute on Particle Physics, eds. L. DeProcel, Ch. Dunwoodie, Stanford, 1993, p. 231, hep-ph/9405376.

[26] W. Porod, Comput. Phys. Commun. 153 (2003) 275 hep-ph/0301101].

[27] K. Desch, J. Kalinowski, G. Moortgat-Pick, K. Rolbiecki and W. J. Stirling, hep-ph/0607104.

[28] Joint LEP2 SUSY Working Group, ALEPH, DELPHI, L3 and OPAL experiments, http://lepsusy.web.cern.ch/lepsusy/Welcome.html.

[29] W. M. Yao et al. [Particle Data Group], J. Phys. G 33 (2006) 1.

[30] A. Bartl, H. Fraas and W. Majerotto, Nucl. Phys. B 278 (1986) 1. 